\title{
Vanishing viscosity method for an optimal control problem of scalar conservation laws in the presence of shocks
}

\author{
Yaobin $\mathrm{Ou}^{1 *}$, Peicheng $\mathrm{Zhu}^{1,2 \dagger}$ \\ ${ }^{1}$ Basque Center for Applied Mathematics (BCAM) \\ Bizkaia Technology Park, Building 500, \\ E-48160 Derio, Spain \\ 2 IKERBASQUE, Basque Foundation for Science \\ E-48011 Bilbao, Spain
}

\begin{abstract}
To reduce the computational cost for optimal control problems of the inviscid Burgers equation in the presence of shocks, Zuazua et al. have developed an alternating descent method, and revisited it by the method of vanishing viscosity. In this article we study theoretically the vanishing viscosity limit of such a problem for 1-D scalar conservation laws with a general nonlinearity. The discontinuities of coefficients in those equations lead to difficulties when taking the limits. Thus we employ the method of matched asymptotic expansions to construct approximate solutions to the smoothed nonlinear, the linearized and the dual problems, respectively. It is then proved that the approximate solutions satisfy the corresponding equations asymptotically, and converge to the solutions of the corresponding inviscid problems with certain convergent rates. The equations for the shock and the variation of its position are derived, and specifically, the latter equation approaches, as a parameter tend to infinity, to the one derived by Bressan and Marson. It is also discussed how to identify the alternating descent directions for the viscous problem.
\end{abstract}

Keywords: Vanishing viscosity limit; optimal control; shock; conservation laws.

AMS Subject Classification: 35L65, 34E05, 35Q93

\section{Introduction}

Optimal control for hyperbolic conservation laws requires a considerable analytical effort and computational expense in practice, is thus a difficult topic. Some methods have been developed in the last years to reduce the computational cost and to render this type of problems affordable. In particular, recently in Ref. [11] Castro, Palacios and Zuazua have developed an "alternating descent method" that takes the possible shock

\footnotetext{
*Corresponding author. E-mail: ou@bcamath.org, ou.yaobin@gmail.com

†E-mail: pczhu@hotmail.com
} 
discontinuities into account, for the optimal control of the inviscid Burgers equation in one space dimension. Further in Ref. [12] this numerical method is also employed to study the optimal control problem of the Burgers equation with small viscosity, with the aid of the Hopf-Cole formula which can be found in Ref. [24, 34], for instance.

In the present article, we study the vanishing viscosity limit of solutions to optimal control problems for 1-D general nonlinear scalar conservation laws in the presence of shocks, which reduces to the vanishing viscosity limits of solutions to the linearized equation (with respect to initial data) of hyperbolic conservation laws, and of reversible solutions to its dual problem. Observing that the discontinuities of coefficients in those equations lead to difficulties when taking to the limit, the method of matched asymptotic expansions is applied to study the convergence. Thus it extends the results in Ref. [12] for Burgers equation to general case. Moreover, certain convergent rates are obtained so that it generalizes the result of James and Sepúlveda (see Ref. [26]) for inverse adjoint equations in some sense. As a result, we justify theoretically that the "alternating descent method" is efficient for the optimal control problem for viscous conservation laws with small viscosity, when limiting solutions to scalar conservation laws have shock discontinuities. Another main contribution of this paper is that, we have derived a new equation for the variation of shock position, which approaches, as a parameter tend to infinity, to the one obtained by Bressan and Marson (see Ref. [9]) which is derived for the invicid Burgers equation in the presence of shocks.

To be more precise, we state the optimal problem as follows. For a given $T>0$, we study the following inviscid problem

$$
\begin{aligned}
u_{t}+(F(u))_{x} & =0, \text { in } \mathbb{R} \times(0, T), \\
u(x, 0) & =u^{I}(x), x \in \mathbb{R} .
\end{aligned}
$$

Here, $F: \mathbb{R} \rightarrow \mathbb{R}$ is a smooth function, and $f$ denotes its derivative in the following context.

Given a target $u^{D} \in L^{2}(\mathbb{R})$ we consider the cost functional to be minimized $J$ : $L^{1}(\mathbb{R}) \rightarrow \mathbb{R}$, defined by

$$
J\left(u^{I}\right)=\int_{\mathbb{R}}\left|u(x, T)-u^{D}(x)\right|^{2} d x,
$$

where $u(x, t)$ is the unique entropy solution to problem (1.1) - (1.2).

We also introduce the set of admissible initial data $\mathcal{U}_{a d} \subset L^{1}(\mathbb{R})$, that we shall define later in order to guarantee the existence of the following optimization problem:

Find $u^{I, \min } \in \mathcal{U}_{a d}$ such that

$$
J\left(u^{I, \min }\right)=\min _{u^{I} \in \mathcal{U}_{a d}} J\left(u^{I}\right) .
$$

Such a problem has been studied in e.g. $[11,12]$ in the case that $F(u)=\frac{u^{2}}{2}$. This is one of the model optimization problems that is often addressed in the context of optimal aerodynamic design, the so-called inverse design problem, see e.g. [19].

The existence of minimizers has been proved in [11]. From a practical point of view it is, however, more important to be able to develop efficient algorithms for computing accurate approximations of discrete minimizers. The most efficient methods to approximate minimizers are the gradient methods. 
But for large complex systems, such as Euler equations in higher dimensions, the existing most efficient numerical schemes (upwind, Godunov, etc.) are not differentiable. In this case, the gradient of the functional is not well defined and there is not a natural and systematic way to compute its variations. Due to this difficulty, it would be natural to explore the possible use of non-smooth optimization techniques. The following two approaches have been developed: The first one is based on automatic differentiation, and the second one is the so-called continuous method consisting of two steps as follows: One first linearizes the continuous system (1.1) to obtain a descent direction of the continuous functional $J$, then takes a numerical approximation of this descent direction with the discrete values provided by the numerical scheme. However this continuous method has to face another major drawback when solutions develop shock discontinuities, as it is the case in the context of the hyperbolic conservation laws like (1.1) we are considering here.

The formal differentiation of the continuous states equation (1.1) yields

$$
\partial_{t}(\delta u)+\partial_{x}(f(u) \delta u)=0, \text { in } \mathbb{R} \times(0, T) .
$$

But this is only justified when the state $u$ on which the variations are being computed, is smooth enough. In particular, it is not justified when the solutions are discontinuous since singular terms may appear on the linearization over the shock location. Accordingly in optimal control applications we also need to take into account the sensitivity for the shock location (which has been studied by many authors, see, e.g. [9, 20, 33]). Roughly speaking, the main conclusion of that analysis is that the classical linearized system for the variation of the solutions must be complemented with some new equations for the sensitivity of the shock position.

To overcome this difficulty, we naturally think of another way, namely, the vanishing viscosity method (as in [12], in which an optimal control problem for the Burgers equation is studied) and add an artificial viscosity term to smooth the inviscid Burgers equation. Equation (1.1) with regularized initial datum then turns out to be

$$
\begin{aligned}
u_{t}+(F(u))_{x} & =\nu u_{x x}, \text { in } \mathbb{R} \times(0, T), \\
\left.u\right|_{t=0} & =g^{\varepsilon} .
\end{aligned}
$$

Note that the Cauchy problem (1.4) - (1.5) is of parabolic type, thus from the standard theory of parabolic equations (see, for instance, Ladyzenskaya et al [28]) we have that the solution $u_{\nu, \varepsilon}$ of this problem is smooth. So the linearized one of eq. (1.4) can be derived easily, which reads

$$
\begin{aligned}
(\delta u)_{t}+(f(u) \delta u)_{x} & =\nu(\delta u)_{x x}, \text { in } \mathbb{R} \times(0, T), \\
\left.\delta u\right|_{t=0} & =h^{\varepsilon} .
\end{aligned}
$$

Here $\nu, \varepsilon$ are positive constants, $\delta u$ denotes the variation of $u$. The initial data $g^{\varepsilon}, h^{\varepsilon}$ will be chosen suitably in Section 3, so that the perturbations of initial data and shock position are taken into account, this renders us that we can select the alternating descent directions in the case of viscous conservation laws.

To solve the optimal control problem, we also make use of the following dual problem, which reads

$$
\begin{aligned}
-p_{t}-f(u) p_{x} & =0, \text { in } \mathbb{R} \times(0, T), \\
p(x, T) & =p^{T}(x), x \in \mathbb{R},
\end{aligned}
$$


here, $p^{T}(x)=u(x, T)-u^{D}(x)$. And we mollify the equation (1.8) and initial data as follows

$$
\begin{aligned}
-p_{t}-f(u) p_{x} & =\nu p_{x x}, \text { in } \mathbb{R} \times(0, T), \\
p(x, T) & =p_{\varepsilon}^{T}(x), x \in \mathbb{R} .
\end{aligned}
$$

Recalling that solutions $u=u(x, t ; \nu, \varepsilon), \delta u=\delta u(x, t ; \nu, \varepsilon)$ to problems $(1.4)-(1.5)$ and (1.6) - (1.7), respectively, are smooth, we see that the quasi-shock (or interfacial) regions, instead of shocks, are formed. Our main task is to investigate the limits as $\nu, \varepsilon$ tend to zero. Then natural questions arise as follows:

1. In order to take into account infinitesimal translations of shock position and infinitesimal perturbations of initial data, and to recover the correct equation for variations of shock and the inviscid limit equations corresponding to (1.10), (1.6) and (1.4) respectively, how should $\nu, \varepsilon$ go to zero? More precisely, can $\nu, \varepsilon$ go to zero independently? Which one goes to zero faster, or the same?

2. Suppose that we can find a suitable relation of $\nu, \varepsilon$ in 1 ). What happens as the two parameters $\nu, \varepsilon$ go to 0 ?

3. To solve the optimal control problem correctly, the states of system (1.3) should be understood as a pair $(\delta u, \delta \varphi)$, where $\delta \varphi$ is the variation of shock position. As $\nu, \varepsilon \rightarrow 0$, is there an equation for $\delta \varphi$ which determines the evolution of $\delta \varphi$ and complements equation (1.3)?

4. Does the alternating descent method work for optimal control problem in the viscous case?

To answer these questions, we shall employ the method of matched asymptotic expansions. Our main results are:

First, in order to construct asymptotic expansions which take into account infinitesimal translations of shock position and infinitesimal perturbations of initial data, the parameters $\nu, \varepsilon$ must satisfy

$$
\varepsilon=\sigma \nu
$$

where $\sigma$ is a given positive constant. This means that $\nu, \varepsilon$ must go to zero at the same order, but speeds may be different. Write $\frac{\varepsilon}{\nu}=\sigma$. Then we assert that if $\sigma>1, \nu$ goes to zero faster than $\varepsilon$, and vice versa.

We now fix $\varepsilon$ which is assumed to be very small. As $\sigma \rightarrow \infty$, namely $\nu \rightarrow 0$, the equation (see equation (3.61)) of variation of shock position differs from the one derived by Bressan and Marson [9], etc., by a term which converges to zero as $\sigma$ tends to infinity, however may be very large if $\sigma$ is small enough. Thus we conclude that

i) The equation derived by Bressan and Marson, which could be recovered by letting $\sigma \rightarrow \infty$ in the equation for $\delta \varphi$ in our paper, is suitable for developing the numerical scheme when $\sigma$ is sufficiently large. In this case, the perturbation of initial data plays a dominant role and the effect due to the artificial viscosity can be omitted.

ii) However, if $\sigma$ is small, then the effect of viscosity must be taken into account while the perturbation of initial data can be neglected, and a corrector should be added.

Second, we make use of matched asymptotic expansions to construct approximations of the solutions to problems $(1.4)-(1.5)$ and (1.10) - (1.11), then prove that they 
converge, respectively, to the entropy solution and the reversible solution of the corresponding inviscid problems, while the solution to problem (1.6) - (1.7) converges to the one that solves (1.3) in the sub-regions away from the shock, and is complemented by an equation i.e. equation (3.61), which governs the evolution of the variation of shock position. The main convergence results are stated in Theorems 3.1 and 4.1.

Third, by using the convergence results, we conclude that for numerical purpose, the alternating descent method is also applicable to optimal control problem in the viscous case, if $\nu$ is sufficiently small, and more efficient than standard method; this method does not work well if $\nu$ is not small, while the standard method is more suitable, see Conclusion 5.1 in Section 5. In practice, $\nu$ is the thickness of interfacial (quasi-shock) region which is usually very small, thus it deserves us to investigate this alternating descent method in the context of viscous conservation laws.

Fourth, using the method of asymptotic expansions we also clarify some formal expansions used frequently in the theory of optimal control, that they are valid only away from shock and when some parameter is not too small. For example, for solution $u_{\nu}$ to problem (1.4) - (1.5) we normally expand it as

$$
u_{\nu}=u+\nu \delta u_{\nu}+O\left(\nu^{2}\right)
$$

where $u$ is usually believed to be the entropy solution to problem (1.1) - (1.2), and $\delta u$ is the variation of $u_{\nu}$, the solution to (1.6) - (1.7). However (1.12) is not correct near shock provided that $\delta u_{\nu}(x, 0)$ is bounded, for instance. From this assumption, we have $\delta u_{\nu}$ is continuous and uniformly bounded by the theory of parabolic equations, moreover $u_{\nu}$ is continuous too, thus it follows from (1.12) that $u$ should be continuous too, but $u$ is normally discontinuous. Therefore, we should understand (1.12) as a multi-scale expansion, and assume that $u=u(x, x / \nu, t), \delta u=\delta u(x, x / \nu, t)$. We shall obtain such an expansion by the method of matched asymptotic expansions.

The new features to the method of asymptotic expansions in this article are mainly as follows:

1. Our expansions for $u_{\nu, \varepsilon}$ and $\delta u_{\nu, \varepsilon}$ are different from the standard ones due to the fact that equations (1.6) and (1.4) are not completely independent, in fact, (1.6) is the linearization of (1.4), so when constructing asymptotic expansions we should take this fact into account and find some compatible conditions for the asymptotic expansions of $u_{\nu, \varepsilon}$ and $\delta u_{\nu, \varepsilon}$.

2. When constructing inner expansions of the solution $p$ to the dual problem, the matching conditions (see (3.103), (3.104) etc.) enable us to show that the approximate solution will approach a constant in a "triangle region", which is occupied by the characteristics forming the shock.

3. We derive the equation of variation of shock location from the outer expansions, but not from the inner expansions as usual, see, e.g. [15]. Our approach is based upon the key observations that the outer expansions converge to their values at the shock, and the quasi-shock region vanishes as $\nu \rightarrow 0$.

4. In contrast with the paper by James and Sepúlveda [26], we obtain a convergent rate for the dual problem, moreover, only need the terminal data to be continuous, instead of $W^{1, \infty} \cap B V$.

We need to introduce some 
Notations: For any $t>0$, we define $Q_{t}=\mathbb{R} \times(0, t) . C(t), C_{a}, \cdots$ denote, respectively, constants depending on $t, a, \cdots$, and $C$ is a universal constant in Sub-Section 4.2.

For a function $f=f(r, t)$ where $r=r_{\nu}(x, t ; \nu): f_{t}$ denotes the partial derivative with respect to $t$ while $(f)_{t}=f_{t}+f_{r} r_{t}$, and so on.

Let $X$ be a Banach space endowed with a norm $\|\cdot\|_{X}$, and $f:[0, T] \rightarrow X$. For any fixed $t$ the $X$-norm of $f$ is denoted by $\|f(t)\|_{X}$, when $X=L^{2}(\mathbb{R})$, we write $\|f(t)\|=\|f(t)\|_{X}$, sometimes the argument $t$ is omitted.

Landau symbols $O(1)$ and $o(1)$. A quantity $f(x, t ; \nu)=o(1)$ means $\|f\|_{L^{\infty}\left(Q_{t}\right)} \rightarrow 0$ as $\nu \rightarrow 0$, and $g(t ; \xi)=o(1)$ implies that $\|g\|_{L^{\infty}(0, t)} \rightarrow 0$ as $\xi \rightarrow \infty$. And $f(x, t ; \nu)=O(1)$ means $\|f\|_{L^{\infty}\left(Q_{t}\right)} \leq C$ uniformly for $\nu \in(0,1]$. We also use the standard notations: $B V(\mathbb{R})\left(B V_{\text {loc }}(\mathbb{R})\right), \operatorname{Lip}(\mathbb{R})\left(\operatorname{Lip}_{\text {loc }}(\mathbb{R})\right)$, are the spaces of the functions of (locally) bounded variations, the (locally) Lipschitz continuous functions in $\mathbb{R}$, respectively.

The remaining parts of this article are as follows: In Section 2, we collect some preliminaries and explain furthermore the motivation of this article. In Section 3, employing the method of matched asymptotic expansions and taking into account the infinitesimal perturbations of the initial datum and the infinitesimal translations of the shock position, we shall construct, the inner and outer expansions, and obtain, by a suitable combination of the two expansions, the approximate solutions to problems (1.4)-(1.5), (1.6)-(1.7), and (1.10)-(1.11). Also the equations for the shock and its variation will be derived. In Section 4 we shall prove the approximate solutions satisfy the corresponding equations asymptotically, and converge, respectively, to those of the inviscid problems in a suitable sense. Finally we discuss the alternating descent method in the context of viscous conservation laws in Section 5, where the convergence results will be used.

\section{Sensitivity analysis: the inviscid case}

A solution to a hyperbolic equation may become singular after certain time, even the initial datum is smooth. Therefore in practical applications it is more interesting to consider optimal control problems in the case that shocks appear. We shall study the optimal control problem for the inviscid equation in the presence of shocks, and we focus on the particular case that solutions have a single shock, however the analysis can be extended to consider more general one-dimensional systems of conservation laws with a finite number of non-interacting shocks, see [8,21].

To develop the alternating descent method for the optimal control problem in presence of shocks, we need to investigate the sensitivity of the states of the system, with respect to perturbations of the initial datum and infinitesimal translations of the shock position. we shall see that equations (1.3) and (1.8) will become much more complicated. This section is devoted to introducing some basic tools needed in the sensivity analysis (see also $[11,12])$.

\subsection{Linearization of the inviscid equation}

Let us firstly introduce the following hypothesis:

(H) Assume that $u$ is a weak entropy solution to (1.1)-(1.2) with a discontinuity along a regular curve $\Sigma=\{(\varphi(t), t) \mid t \in[0, T)\}$ which is Lipschitz continuous outside $\Sigma$. The 
Rankine-Hugoniot condition is satisfied on $\Sigma$ :

$$
\varphi^{\prime}(t)[u]_{\varphi(t)}=[F(u)]_{\varphi(t)} .
$$

Hereafter, we denote the jump at $x=\varphi(t)$ of a piecewise smooth function $f=f(x, t)$ by $[f]_{\varphi(t)}=f(\varphi(t)+0, t)-f(\varphi(t)-0, t)$, for any fixed $t$.

Note that $\Sigma$ divides $Q_{T}=\mathbb{R} \times(0, T)$ into two subdomains $Q^{-}$and $Q^{+}$, to the left and to the right of $\Sigma$ respectively. see Figure 1 .

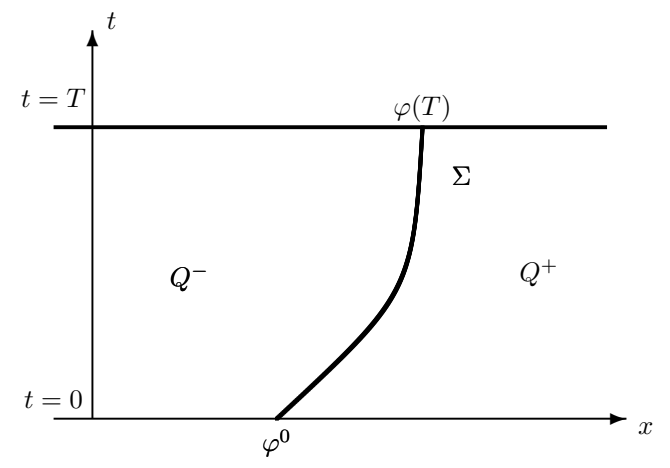

Figure 1: Subdomains $Q^{-}$and $Q^{+}$.

As analyzed in [11], to deal correctly with the optimal control and design problems, the state of the system should be viewed as the pair $(u, \varphi)$ consisting of the solution of (1.1) and the shock position $\varphi$. The pair $(u, \varphi)$ satisfies

$$
\begin{aligned}
u_{t}+(F(u))_{x} & =0, \text { in } Q^{+} \cup Q^{-}, \\
\varphi^{\prime}(t)[u]_{\varphi(t)} & =[F(u)]_{\varphi(t)}, t \in(0, T), \\
\varphi(0) & =\varphi^{I}, \\
u(x, 0) & =u^{I}(x), x \in\left\{x<\varphi^{I}\right\} \cup\left\{x>\varphi^{I}\right\} .
\end{aligned}
$$

We also need to analyze the sensitivity of $(u, \varphi)$ with respect to perturbations of the initial datum, especially, with respect to $\delta u^{I}$ and $\delta \varphi^{I}$ which are variations of the initial profile $u^{I}$ and of the shock position $\varphi^{I}$, respectively. To be precise, we first need to introduce the functional framework based on the generalized tangent vectors introduced in $[8]$.

Definition 2.1 Let $v: \mathbb{R} \rightarrow \mathbb{R}$ be a piecewise Lipschitz continuous function with a single discontinuity at $y \in \mathbb{R}$. We define $\Sigma_{v}$ as the family of all continuous paths $\gamma:\left[0, \varepsilon_{0}\right] \rightarrow$ $L^{1}(\mathbb{R})$ with

(1) $\gamma(0)=v$ and $\varepsilon_{0}>0$ possibly depending on $\gamma$.

(2) For any $\varepsilon \in\left[0, \varepsilon_{0}\right]$ the functions $v^{\varepsilon}=\gamma(\varepsilon)$ are piecewise Lipschitz with a single discontinuity at $x=y^{\varepsilon}$ depending continuously on $\varepsilon$ and there exists a constant $L$ independent of $\varepsilon \in\left[0, \varepsilon_{0}\right]$ such that

$$
\left|v^{\varepsilon}(x)-v^{\varepsilon}\left(x^{\prime}\right)\right| \leq L\left|x-x^{\prime}\right|,
$$

whenever $y^{\varepsilon} \notin\left[x, x^{\prime}\right]$. 
Furthermore, we define the set $T_{v}$ of generalized tangent vectors of $v$ as the space of $(\delta v, \delta y) \in L^{1} \times R$ for which the path $\gamma_{(\delta v, \delta y)}$ given by

$$
\gamma_{(\delta v, \delta y)}(\varepsilon)= \begin{cases}v+\varepsilon \delta v+[v]_{y} \chi_{[y+\varepsilon \delta y, y]} & \text { if } \quad \delta y<0, \\ v+\varepsilon \delta v-[v]_{y} \chi_{[y, y+\varepsilon \delta y]} & \text { if } \quad \delta y>0,\end{cases}
$$

satisfies $\gamma_{(\delta v, \delta y)} \in \Sigma_{v}$.

Finally, we define the relation of equivalence $\sim$ in $\Sigma_{v}$ by

$$
\gamma \sim \gamma^{\prime} \text { if and only if } \lim _{\varepsilon \rightarrow 0} \frac{\left\|\gamma(\varepsilon)-\gamma^{\prime}(\varepsilon)\right\|_{L^{1}}}{\varepsilon}=0,
$$

and we say that a path $\gamma \in \Sigma_{v}$ generates the generalized tangent vector $(\delta v, \delta y) \in T_{v}$ if $\gamma$ is equivalent to $\gamma_{(\delta v, \delta y)}$.

Remark 2.1. The path $\gamma_{(\delta v, \delta y)} \in \Sigma_{v}$ represents, at first order, the variation of a function $v$ by adding a perturbation function $\varepsilon \delta v$ and by shifting the discontinuity by $\varepsilon \delta y$.

Note that, for a given $v$ (piecewise Lipschitz continuous function with a single discontinuity at $y \in \mathbb{R})$, the associated generalized tangent vectors $(\delta v, \delta y) \in T_{v}$ are those pairs for which $\delta v$ is piecewise Lipschitz continuous with a single discontinuity at $x=y$.

Now we assume that the initial datum $u^{I}$ is Lipschitz continuous to both sides of a single discontinuity at $x=\varphi^{I}$, and consider a generalized tangent vector $\left(\delta u^{I}, \delta \varphi^{I}\right) \in$ $L^{1}(\mathbb{R}) \times \mathbb{R}$. Let $u^{I, \varepsilon} \in \Sigma_{u^{I}}$ be a path which generates $\left(\delta u^{I}, \delta \varphi^{I}\right)$. For sufficiently small $\varepsilon$ the solution $u^{\varepsilon}$ of problem (2.1) - (2.4) is Lipschitz continuous with a single discontinuity at $x=\varphi^{\varepsilon}(t)$, for all $t \in[0, T]$. Thus $u^{\varepsilon}$ generates the generalized tangent vector $(\delta v, \delta \varphi) \in$ $L^{1}(\mathbb{R}) \times \mathbb{R}$. Then it is proved in [9] that $(\delta u, \delta \varphi)$ satisfies the linearized system

$$
\begin{aligned}
(\delta u)_{t}+(f(u) \delta u)_{x} & =0, \text { in } Q^{+} \cup Q^{-} \\
(\delta \varphi)^{\prime}(t)[u]_{\varphi(t)} & +\delta \varphi(t)\left(\varphi^{\prime}(t)\left[u_{x}\right]_{\varphi(t)}-\left[f(u) u_{x}\right]_{\varphi(t)}\right) \\
& =[f(u) \delta u]_{\varphi(t)}-\varphi^{\prime}(t)[\delta u]_{\varphi(t)}, t \in(0, T), \\
\delta \varphi(0) & =\delta \varphi^{I} \\
\delta u(x, 0) & =\delta u^{I}(x), x \in\left\{x<\varphi^{I}\right\} \cup\left\{x>\varphi^{I}\right\} .
\end{aligned}
$$

Remark 2.2. In this way, we can obtain formally the expansion:

$$
\left(u^{\varepsilon}, \varphi^{\varepsilon}\right)=(u, \varphi)+\varepsilon(\delta u, \delta \varphi)+O\left(\varepsilon^{2}\right) .
$$

Unfortunately, this expansion is, in general, not true, as we explained in the introduction. For instance, suppose that $\delta u$ is bounded and $u^{\varepsilon}$ is continuous. From (2.9) we conclude that $u^{\varepsilon}$ converges to $u$ uniformly, whence $u$ should be continuous too. But this is not true in general. Thus we should assume $(u, \delta u)=(u, \delta u)(x, x / \varepsilon, t)$, a multi-scale expansion.

Remark 2.3. In Section 3, we shall see that equation (2.6) can not be, as expected, recovered as the viscosity $\nu$ tends to zero. Instead, it is changed to

$$
\begin{aligned}
{[u]_{\varphi(t)} \delta \varphi^{\prime}(t)=} & \delta \varphi(t)\left(-\left[u_{x}\right]_{\varphi(t)} \varphi^{\prime}(t)+\left[f(u) u_{x}\right]_{\varphi(t)}\right) \\
& +\left(-[\delta u]_{\varphi(t)} \varphi^{\prime}(t)+[f(u) \delta u]_{\varphi(t)}\right) \\
& +\frac{1}{\sigma}\left(\left[u_{x}\right]_{\varphi(t)}-\left([w]_{\varphi(t)} \varphi^{\prime}(t)-[f(u) w]_{\varphi(t)}\right)\right),
\end{aligned}
$$


in which a corrector (the term involving $\frac{1}{\sigma}$ ) is added. Here $w$ is a function which will be constructed by asymptotic expansion and has limits as $x \rightarrow \pm \varphi(t)$ for $t \in(0, T)$.

\subsection{Sensitivity in presence of shocks}

To study the sensitivity, in the presence of shocks, of $J$ with respect to variations associated with the generalized tangent vector, we define an appropriate generalization of the Gateaux derivative.

Definition 2.2 (Ref. [8]) Let $J: L^{1}(\mathbb{R}) \rightarrow \mathbb{R}$ be a functional and $u^{I} \in L^{1}(\mathbb{R})$ be a Lipschitz continuous initial datum with a discontinuity at $x=\varphi^{I}$ for which the solution of (1.1) satisfies hypothesis $(H) . J$ is Gateaux differentiable at $u^{I}$ in a generalized sense if for any generalized tangent vector $\left(\delta u^{I}, \delta \varphi^{I}\right)$ and any family $u^{I, \varepsilon} \in \Sigma_{u^{I}}$ associated to $\left(\delta u^{I}, \delta \varphi^{I}\right)$ the following limit exists,

$$
\delta J=\lim _{\varepsilon \rightarrow 0} \frac{J\left(u^{I, \varepsilon}\right)-J\left(u^{I}\right)}{\varepsilon} .
$$

Moreover, it depends only on $\left(u^{I}, \varphi^{I}\right)$ and $\left(\delta u^{I}, \delta \varphi^{I}\right)$, i.e. it does not depend on the particular family $u^{I, \varepsilon}$ which generates $\left(\delta u^{I}, \delta \varphi^{I}\right)$. The limit is the generalized Gateux derivative of $J$ in the direction $\left(\delta u^{I}, \delta \varphi^{I}\right)$.

Then we have the proposition which characterizes the generalized Gateaux derivative of $J$ in terms of the solution to the associated adjoint system.

Proposition 2.1 Assume that $u^{D}$ is continuous at $x=\varphi(T)$. The Gateaux derivative of $J$ can be written as follows:

$$
\delta J=\int_{\left\{x<\varphi^{I}\right\} \cup\left\{x>\varphi^{I}\right\}} p(x, 0) \delta u^{I}(x) d x+q(0)\left[u^{I}\right]_{\varphi^{I}} \delta \varphi^{I},
$$

where the adjoint state pair $(p, q)$ satisfies the system

$$
\begin{aligned}
-\partial_{t} p-f(u) \partial_{x} p & =0, \text { in } Q^{-} \cup Q^{+}, \\
{[p]_{\Sigma} } & =0, \\
q(t) & =p(\varphi(t), t), t \in(0, T), \\
q^{\prime}(t) & =0, t \in(0, T), \\
p(x, T) & =u(x, T)-u^{D}(x), x \in\{x<\varphi(T)\} \cup\{x>\varphi(T)\}, \\
q(T) & =\frac{\left[F\left(u(x, T)-u^{D}(x)\right)\right]_{\varphi(T)}}{[u]_{\varphi(T)}} .
\end{aligned}
$$

Remark 2.4. The backward system (2.11)-(2.16) has a unique solution. We can solve it in the following way: We first define the solution $q$ on the shock $\Sigma$ from the condition $q^{\prime}=0$, with the given final value $q(T)$. Then this determines the value of $p$ along the shock. We then can propagate this information, together with the datum of $p$ at time $T$ to both sides of $\varphi(T)$, by characteristics. As both systems (1.1) and (2.11) have the same characteristics, any point $(x, t) \in \mathbb{R} \times(0, T)$ is reached backwards in time by a unique characteristics line coming either from the shock $\Sigma$ or the final data at $(x, T)$ (see Fig. 2). The solution obtained in this way coincides with the reversible solutions introduced in [6]. 

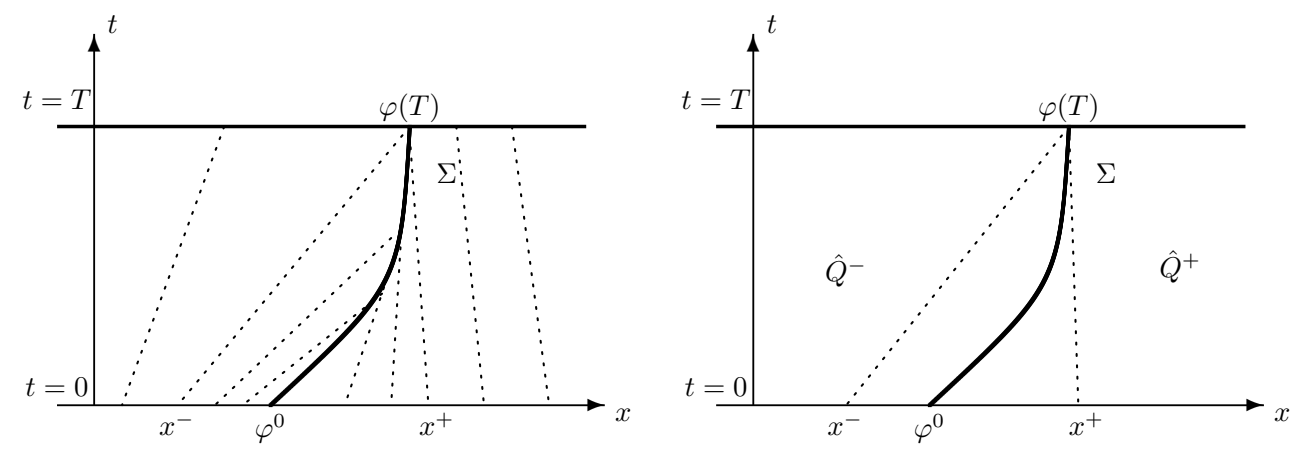

Figure 2: Characteristic lines entering on a shock(left) and subdomains $\hat{Q}^{-}$and $\hat{Q}^{+}$(right).

In Figure 2, we have used the following notations:

$$
x^{-}=\varphi(T)-u^{-}(\varphi(T)) T, x^{+}=\varphi(T)-u^{+}(\varphi(T)) T,
$$

and

$$
\begin{aligned}
& \hat{Q}^{-}=\left\{(x, t) \in \mathbb{R} \times(0, T) \text { such that } x<\varphi(T)-u^{-}(\varphi(T)) t\right\} \\
& \hat{Q}^{+}=\left\{(x, t) \in \mathbb{R} \times(0, T) \text { such that } x>\varphi(T)-u^{+}(\varphi(T)) t\right\} .
\end{aligned}
$$

Remark 2.5. We shall construct a solution to system (2.11)-(2.16) in the following manner: We approximate the terminal datum $P^{T}$ by the functions $p^{T}(n=1,2, \cdots)$ which satisfy:

$p^{T}(n \in \mathbb{Z})$ are locally Lipschitz continuous, uniformly bounded in $W_{\text {loc }}^{1,1}(\mathbb{R})$ such that

$$
p^{T}(\cdot, T) \rightarrow P^{T}(\cdot)=u(\cdot, T)-u^{D}(\cdot) \text { in } L_{\mathrm{loc}}^{1}(\mathbb{R}),
$$

and

$$
p^{T}(\varphi(T), T)=\frac{\left[F\left(u(x, T)-u^{D}(x)\right)\right]_{\varphi(T)}}{[u]_{\varphi(T)}} .
$$

Then we first take the limit of solutions $p_{\nu, n}$ of (1.10) with initial data $p^{T}$, as $\nu \rightarrow 0$ to obtain the solution $p_{n}$ of

$$
\begin{aligned}
-\partial_{t} p-f(u) \partial_{x} p & =0, \text { in } \mathbb{R} \times(0, T), \\
p(x, T) & =p^{T}(x), \text { in } \mathbb{R},
\end{aligned}
$$

the so-called reversible solution. These solutions can be characterized by the fact that they take the value $p_{n}(\varphi(T), T)$ in the whole region occupied by the characteristics that meet the shock. Thus in particular they satisfy the equations (2.12)-(2.14) and (2.16). Moreover, $p_{n} \rightarrow p$ as $n \rightarrow \infty$, and $p$ takes a constant value in the region occupied by the characteristics that meet the shock. Note that by construction, this constant is the same for all $p_{n}$ in this region. Thus this limit solution $p$ coincides with the solution of system (2.11)-(2.16).

In this article, we shall apply the method of matched asymptotic expansions to justify these convergence results. 


\subsection{The method of alternating descent directions: Inviscid case}

We shall present, in this sub-section, the main ideas of the alternating descent method which is introduced in [11] for the inviscid Burgers equation. The classification of the generalized tangent vectors into two cases is motivated by the following proposition.

Proposition 2.2 Assume that we restrict the set of paths in $\Sigma_{u^{I}}$ to those for which the associated generalized tangent vectors $\left(\delta u^{I}, \delta \varphi^{I}\right) \in T_{u^{I}}$ satisfy

$$
\delta \varphi^{I}=\frac{\int_{x^{-}}^{\varphi^{I}} \delta u^{I} d x+\int_{\varphi^{I}}^{x^{+}} \delta u^{I} d x}{\left[u^{I}\right]_{\varphi^{I}}} .
$$

Then, the solution $(\delta u, \delta \varphi)$ of problem $(2.5)-(2.8)$ satisfies $\delta \varphi(T)=0$ and the general Gateaux derivative of $J$ can be written as follows:

$$
\delta J=\int_{\left\{x<x^{-}\right\} \cup\left\{x>x^{+}\right\}} p(x, 0) \delta u^{I}(x) d x,
$$

where the adjoint state pair $p$ satisfies the system

$$
\begin{aligned}
-\partial_{t} p-f(u) \partial_{x} p & =0, \text { in } Q^{-} \cup Q^{+}, \\
p(x, T) & =u(x, T)-u^{D}(x), x \in\{x<\varphi(T)\} \cup\{x>\varphi(T)\} .
\end{aligned}
$$

Analogously, when considering paths in $\Sigma_{u^{I}}$ for which the associated generalized tangent vectors $\left(\delta u^{I}, \delta \varphi^{I}\right) \in T_{u^{I}}$ satisfy that $\delta u^{I}=0$, then $\delta u(x, T)=0$ and the general Gateaux derivative of $J$ in the direction $\left(\delta u^{I}, \delta \varphi^{I}\right)$ can be written as

$$
\delta J=-\frac{\left[F\left(u(x, T)-u^{D}(x)\right)\right]_{\varphi(T)}}{[u]_{\varphi(T)}}\left[u^{I}\right]_{\varphi^{I}} \delta \varphi^{I} .
$$

Remark 2.6. Formula (2.20) establishes a simplified expression for the generalized Gateaux derivative of $J$ in the direction $\left(\delta u^{I}, \delta \varphi^{I}\right)$ without considering the variation of shock position at $t=T$. This direction is characterized by formula (2.19) which determines the infinitesimal displacement of the shock position $\delta \varphi^{I}$ in terms of the variation of $u^{I}$ to both sides of $x=\delta \varphi^{I}$. Note, in particular, that for any value $\delta u^{I}$ to both sides of the jump $\varphi^{I}$, the infinitesimal translation $\delta \varphi^{I}$ corresponds uniquely to the shock position without variation. We see that formula (2.20) holds even if $u^{D}$ is discontinuous at $x=\varphi(T)$, since we are dealing with a subspace of generalized tangent vectors satisfying $\delta \varphi(T)=0$ and the Gateaux derivative of $J$, reduced to this subspace, is well defined.

Note also that system $(2.21)-(2.22)$ does not allow to determine $p$ in $(\mathbb{R} \times[0, T]) \backslash\left(\hat{Q}^{-} \cup\right.$ $\left.\hat{Q}^{+}\right)$, i.e. the region under the influence of the shock by the characteristic lines emanating from it. However, the value of $p$ in this region is not required to evaluate the generalized Gateaux derivative in (2.20). Analogously, formula (2.23) provides uniquely a simplified expression of the generalized Gateaux derivative of $J$ in the direction $\left(0, \delta \varphi^{I}\right)$.

Note that the results in Proposition 2.2 suggest the following decomposition of the set of generalized tangent vectors:

$$
T_{u^{I}}=T_{u^{I}}^{1} \oplus T_{u^{I}}^{2},
$$


where $T_{u^{I}}^{1}$ consists of those $\left(\delta u^{I}, \delta \varphi^{I}\right)$ for which identity (2.19) holds, and $T_{u^{I}}^{2}$ consists of the ones for which $\delta u^{I}=0$. Thus this provides two classes of descent directions for $J$ at $u^{I}$. In principle they are not optimal steepest descent directions, but they both have three important properties:

i) They are both descent directions.

ii) They allow to separate the design of the profile from the shock location.

iii) They are exactly the generalized gradients that do not increase the complexity of the structure of initial data.

When considering generalized tangent vectors belonging to $T_{u^{I}}^{1}$, we can choose as descent direction,

$$
\delta u^{I}= \begin{cases}-p(x, 0) & \text { if } x<x^{-}, \\ -\lim _{x \rightarrow x^{-}}, x<x^{-} p(x, 0) & \text { if } x^{-}<x<\varphi^{I}, \\ -\lim _{x \rightarrow x^{+}, x>x^{+}} p(x, 0) & \text { if } \varphi^{I}<x<x^{+}, \\ -p(x, 0) & \text { if } x^{+}<x,\end{cases}
$$

and

$$
\delta \varphi^{I}=-\frac{\int_{x^{-}}^{\varphi^{I}} p(x, 0) d x+\int_{\varphi^{I}}^{x^{+}} p(x, 0) d x}{[u]_{\varphi^{I}}}
$$

while for $T_{u^{I}}^{2}$ a good choice is:

$$
\delta u^{I}=0, \delta \varphi^{I}=\left[F\left(u(x, T)-u^{D}(x)\right)\right]_{\varphi(T)} \frac{[u(\cdot, T)]_{\varphi(T)}}{\left[u^{I}\right]_{\varphi^{I}}} .
$$

In (2.26) the value of $\delta \varphi^{I}$ in the interval $\left(x^{-}, x^{+}\right)$does not affect the generalized Gateaux derivative in (2.20) under the condition that $\delta \varphi^{I}$ is chosen exactly as indicated (otherwise the shock would move and this would produce an extra term on the derivative of the functional $J$ ). We have chosen simply the constant value that preserves the Lipschitz continuity of $\delta u^{I}$ at $x=x^{-}$and $x=x^{+}$, but not necessarily at $x=\delta \varphi^{I}$. Other choices would also provide descent directions for $J$ at $u^{I}$, but yield the same Gateaux derivative according to $(2.20)$.

This allows us to set up a strategy to obtain descent directions for $J$ at $u^{I}$ in $T_{u^{I}}$.

Based upon these studies, an alternating descent method for the optimal control of the Burgers equation can thus be developed, by applying in each step of the descent, the following two sub-steps:

i) Use generalized tangent vectors that move the shock to search its optimal placement.

ii) Use generalized tangent vectors to modify the value of the solution at time $t=T$ to both sides of the discontinuity, leaving the shock location unchanged.

For more details, we refer the reader to [11], and a number of numerical experiments show that this method is much more robust and efficient than the usual ones.

In this article, motivated by the strategy for obtaining descent directions for $J$ in the inviscid case, we will carry out, in Section 5, this procedure for viscous conservation laws provided that the parameter $\nu$ is sufficiently small. 
We now turn to discuss the existence of minimizers of the functional $J$, depending on a small parameter that comes from the solutions to the viscous problems, over suitable admissible set and their limit as the small parameter tends to zero. Let us now introduce the set of admissible initial data $\mathcal{U}_{a d} \subset L^{1}(\mathbb{R})$, which is

$$
\mathcal{U}_{a d}=\left\{f \in L^{\infty}(\mathbb{R}) \mid \operatorname{supp}(f) \subset K,\|f\|_{L^{\infty}(\mathbb{R})} \leq C\right\},
$$

where $K \subset \mathbb{R}$ is a bounded interval and $C>0$ a constant. We shall see later this choice guarantees the existence of minimizers for the following optimization problem:

Find $u^{I, \min } \in \mathcal{U}_{a d}$ such that

$$
J\left(u^{I, \min }\right)=\min _{u^{I} \in \mathcal{U}_{a d}} J\left(u^{I}\right) .
$$

To make the dependence on the viscosity parameter $\nu$ more explicit the functional $J$ will be denoted by $J_{\nu}$, although its definition is the same as that of $J$. Similarly we now consider the same minimization problem for the viscous model (1.4):

Find $u^{I, \min } \in \mathcal{U}_{a d}$ such that

$$
J_{\nu}\left(u^{I, \min }\right)=\min _{u^{I} \in \mathcal{U}_{a d}} J_{\nu}\left(u^{I}\right)
$$

For the existence of minimizers of the functionals $J$ and $J_{\nu}$, and the conclusion that the minimizers of the viscous problem $(\nu>0)$ converge to a minimizer of the inviscid problem as the viscosity goes to zero, we refer to a recent work [12] for the vanishing viscosity method for the Burgers equation. In that paper, the following theorems are proved.

Theorem 2.1 (Existence of Minimizers) Assume that $\mathcal{U}_{a d}$ is defined in $(2.28)$ and $u^{D} \in$ $L^{2}(\mathbb{R})$. Then the minimization problem $(2.29)$ and $(2.30)$ have at least one minimizer $u^{I, \min } \in \mathcal{U}_{a d}$.

Theorem 2.2 Any accumulation point as $\nu \rightarrow 0$ of $u_{\nu}^{I, \text { min }}$, the minimizers of (2.30), with respect to the weak topology in $L^{2}$, is a minimizer of the continuous problem (2.29).

Note that for any positive $\nu$, the solutions $\delta u, p$ of equations (1.6) and (1.10) are smooth, thus the Gateaux derivative of the functional $J$ is as follows

$$
\delta J=<\delta J\left(u^{I}\right), \delta u^{I}>=\int_{\mathbb{R}} p(x, 0) \delta u^{I}(x) d x
$$

where the adjoint state $p=p_{\nu}$ is the solution to (1.10) with terminal datum $p(x, T)=$ $u(x, T)-u^{D}$.

Unlike in the inviscid one, the adjoint state doesn't contain the adjoint shock variable since the state does not present shocks. Similarly, the derivative of $J$ has also only one component. According to this, the straightforward application of a gradient method for the optimization of $J$ would lead, in each step of the iteration, to make use of the variation pointing in the direction

$$
\delta u^{I}=-p(x, 0)
$$


where $p=p_{\nu}$ is the solution to the viscous dual problem. So the alternating method is considerably simplified. But, when processing this way, we would not be exploiting the possibilities that the alternate descent method provides. Therefore, we take into account the effects of possible infinitesimal perturbations of initial datum and also infinitesimal translations, and use variations of the form

$$
u_{\varepsilon}^{I}(x)=u_{\varepsilon}^{I}\left(x+\varepsilon \delta \varphi^{I}\right)+\varepsilon \delta u_{\varepsilon}^{I}(x),
$$

where, $\varphi^{I}$ stands for a reference point on the profile of $u^{I}$, not necessary a point of discontinuity. When $u^{I}$ has a point of discontinuity, $\varphi^{I}$ could be its location and $\delta \varphi^{I}$ an infinitesimal variation of it. However $\varphi^{I}$ could also be another singular point on the profile of $u^{I}$, as, for instance, an extremal point or a point where the gradient of $u^{I}$ is large, namely a smeared discontinuity. By a Taylor expansion, (2.33) can be rewritten in the following form

$$
u_{\varepsilon}^{I}(x)=u^{I}(x)+\varepsilon\left(\delta \varphi^{I} u_{x}^{I}(x)+\delta u^{I}(x)\right)+O\left(\varepsilon^{2}\right) .
$$

This indicates that the result of these combined variations $\left(\delta u^{I}, \delta \varphi^{I}\right)$ is equivalent to a classical variation in the direction of $\delta \varphi^{I} u_{x}^{I}+\delta u^{I}$. We find that the effect of a small $\delta \varphi^{I}$ can be amplified by a large gradient $\delta u^{I}$.

As we will see in the next section, (2.33) and (2.34) give us some hints on how to construct outer expansions that include the effects of possible infinitesimal perturbations of initial datum and infinitesimal translations.

\section{Matched asymptotic expansions and approximate solu- tions}

In this section, we are going to apply the method of matched asymptotic expansions to construct inner and outer expansions. Then we get the approximate solutions by a suitable combination of them. Firstly, we derive the outer expansions. For the purpose of sensitivity analysis of the states of the system, we add infinitesimal perturbations to the initial datum and infinitesimal translations to the shock position, and make use of Taylor expansions. These operations make it possible to derive the equations for the shock and its variation.

In what follows, for simplicity we introduce

$$
v=\delta u, \psi=\delta \varphi
$$

We consider uniform asymptotic expansions of solutions to the following problems.

$$
\begin{aligned}
u_{t}+(F(u))_{x} & =\nu u_{x x}, \text { in } \mathbb{R} \times(0, T), \\
\left.u\right|_{t=0} & =g^{\varepsilon}, \\
v_{t}+(f(u) v)_{x} & =\nu v_{x x}, \text { in } \mathbb{R} \times(0, T), \\
\left.v\right|_{t=0} & =h^{\varepsilon}, \\
-p_{t}-f(u) p_{x} & =\nu p_{x x}, \text { in } \mathbb{R} \times(0, T), \\
\left.p\right|_{t=T} & =p_{\epsilon}^{T} .
\end{aligned}
$$


Equation (3.1) is the usual conservation law with viscosity, (3.3) is its linearized equation, and (3.7) is the dual equation of (3.3). Problem (3.5) - (3.6) is not well-posed suppose we solve it forward. For convenience, we make a transformation of $t$ variable, i.e. $s=T-t$, and let $p(x, t)=\hat{p}(x, s)$, then this problem is reduced to

$$
\begin{aligned}
\hat{p}_{s}-f(u) \hat{p}_{x} & =\nu \hat{p}_{x x}, \text { in } \mathbb{R} \times(0, T), \\
\left.p\right|_{s=0} & =p_{\epsilon}^{T} .
\end{aligned}
$$

This is now a standard Cauchy problem for a parabolic equation. To avoid too many notations, we still denote $\hat{p}(x, s)$ by $p(x, s)$.

We make the following assumptions for initial/final data and nonlinearity: Assumptions. A1)

The function $F$ is smooth.

A2) Let $n \in \mathbb{N}, g_{0}=u^{I}, h_{0}=\left.\delta u\right|_{t=0}$, and let $p^{T}$ be the initial data to (1.8). Assume that $g_{0}, h_{0} \in L^{1}(\mathbb{R}) \cap L^{\infty}(\mathbb{R}), g_{0 x} \in L^{1}(\mathbb{R}), p^{T} \in L^{1}(\mathbb{R}) \cap C_{\text {loc }}(\mathbb{R}) \cap L^{\infty}(\mathbb{R})$, and that $g_{0 x}, h_{0}$ have only a shock at $\varphi^{I}$. There exist smooth functions $g^{\varepsilon}, h^{\varepsilon}, p_{\varepsilon}^{T} \in C_{0}^{\infty}(\mathbb{R})$, such that

$$
g^{\varepsilon}, h^{\varepsilon}, p_{\varepsilon}^{T} \rightarrow g_{0}, h_{0}, p_{n}^{T}
$$

in $L^{2}(\mathbb{R})$, for example, as $\varepsilon \rightarrow 0$, respectively.

A3) Assume further that Oleinik's one-sided Lipschitz condition (OSLC) is satisfied, i.e.

$$
(f(u(x, t))-f(u(y, t)))(x-y) \leq \alpha(t)(x-y)^{2}
$$

for almost every $x, y \in \mathbb{R}$ and $t \in(0, T)$, where $\alpha \in L^{1}(0, T)$.

\subsection{Outer expansions}

An outer expansion is valid (by this word we mean that the expansion satisfies the equation exactly or with a small error) outside the interfacial (or quasi-shock) region, and is a series of the following form

$$
\eta=\eta(x, t, \nu)=\eta_{0}(x, t)+\nu \eta_{1}(x, t)+\nu^{2} \eta_{2}(x, t)+\cdots,
$$

or

$$
\eta=\eta(x, t, \nu)=\eta^{0}(x, t)+\nu \eta^{1}(x, t)+\nu^{2} \eta^{2}(x, t)+\cdots,
$$

where $\eta, \eta_{0}, \eta^{0}, \eta_{1}, \eta^{1}, \cdots$ depend on variables $x, t$ (but, do not depend on fast variable $r_{\nu}$ that is of order $O\left(\nu^{-1}\right)$ and is defined in the beginning of sub-Section 3.3) and will be replaced by $u, u_{0}, u^{0}, u_{1}, u^{1}, \cdots$, respectively. So we expand the initial data $h^{\varepsilon}, g^{\varepsilon}, p_{n, \varepsilon}^{T}$. Hereafter, we use "..." to denote the remainder which is of higher order of the small parameter $\nu$, thus can be omitted.

The construction of outer expansions consists of three parts. 
Part 1. Outer expansions of $u$. We start with the construction of outer expansion of $u$. The following conventions will apply throughout this sub-section: $u^{i}, u_{j}^{i}$ denote an unknown function with indices $i$ and $i, j$, respectively, where the $i, j$ are non-negative integers. The $k$-th power of $u^{i}$ (res. $u_{j}^{i}$ ) is denoted by $\left(u^{i}\right)^{k}\left(\right.$ res. $\left.\left(u_{j}^{i}\right)^{k}\right)$. However, for numbers, such as the parameters $\nu$, $\varepsilon$, we still use the notation $\nu^{i}$ to stand for the $i$-th power of $\nu$.

We now expand the initial data as follows

$$
\begin{aligned}
h^{\varepsilon} & =h_{0}(x)+\varepsilon h_{1}(x)+\varepsilon^{2} h_{2}(x)+\cdots, \\
p_{\epsilon}^{T} & =p_{0}^{T}(x)+\varepsilon p_{1}^{T}(x)+\varepsilon^{2} p_{2}^{T}(x)+\cdots,
\end{aligned}
$$

and the nonlinear terms as

$$
\begin{aligned}
F(\eta) & =F\left(\eta_{0}\right)+\nu f\left(\eta_{0}\right) \eta_{1}+\nu^{2}\left(f\left(\eta_{0}\right) \eta_{2}+\frac{1}{2} f^{\prime}\left(\eta_{0}\right)\left(\eta_{1}\right)^{2}\right)+\cdots \\
f(\eta) & =f\left(\eta_{0}\right)+\nu f^{\prime}\left(\eta_{0}\right) \eta_{1}+\nu^{2}\left(f^{\prime}\left(\eta_{0}\right) \eta_{2}+\frac{1}{2} f^{\prime \prime}\left(\eta_{0}\right)\left(\eta_{1}\right)^{2}\right)+\cdots
\end{aligned}
$$

which will be used whenever we deal with the nonlinear term.

However, in order to analyze the sensitivity of the states with respect to perturbations of the initial data and the shock position, the initial datum $g^{\varepsilon}$ will be expanded in a special manner, see (3.36). For the same purpose, we construct the outer expansions of solution $u$ to equation (3.1) with (3.14) in the following way: It is easy to see that the solution $u$ depends on two parameters $\nu, \varepsilon$, so we write

$$
u=u(x, t ; \nu, \varepsilon)
$$

and define

$$
x_{\varepsilon}=x+\varepsilon(\nu) \psi(t),
$$

where $\varepsilon=\varepsilon(\nu)$ is a function to be determined. We remark that $\psi(t)$ is indeed the same as $-\xi_{\alpha}$ in Bressan \& Marson [9], therefore there would be negative signs before $\psi^{\prime}(t)$ and $\psi(t)$ in the jump condition for the linearized equation in our paper. Making a transformation of variable $x \rightarrow x_{\varepsilon}$, we obtain a new function

$$
\hat{u}=\hat{u}\left(x_{\varepsilon}, t ; \nu, \varepsilon\right):=u\left(x_{\varepsilon}-\varepsilon(\nu) \psi(t), t ; \nu, \varepsilon\right)=u(x, t ; \nu, \varepsilon) .
$$

For the initial data, we make the same transformation of variable, i.e. (3.18) with $t=0$, and have

$$
g^{\varepsilon}(x)=\hat{g}_{0}\left(x_{\varepsilon}\right)+\varepsilon \hat{g}_{1}\left(x_{\varepsilon}\right)+\varepsilon^{2} \hat{g}_{2}\left(x_{\varepsilon}\right)+\cdots .
$$

Here, we defined $\hat{g}_{i}\left(x_{\varepsilon}\right)=g_{i}\left(x_{\varepsilon}-\varepsilon \psi(0)\right)$ with $i=0,1,2$.

Due to the fact that $u$ depends on two parameters, the outer expansion of $u$ is constructed in two steps so that it has the form (3.12).

Step 1. Firstly we expand $\hat{u}\left(x_{\varepsilon}, t ; \nu, \varepsilon\right)$ in terms of $\varepsilon$ and $(\eta, t)=\left(x_{\varepsilon}, t\right)$ as follows, now $x_{\varepsilon}$ is regarded as an independent variable:

$$
\hat{u}=u^{0}+\varepsilon u^{1}+\varepsilon^{2} u^{2}+\cdots,
$$


where $u^{i}=u^{i}\left(x_{\varepsilon}, t ; \nu\right)$, for $i=0,1,2, \cdots$, and we find, by using the rescaling technique and testing different ansatzs, that in order to get asymptotic expansions there must hold

$$
\varepsilon=\sigma \nu
$$

Step 2. We further expand $u^{i}$ near $(x, t)$ in terms of $\nu$ in the following way. For $i=0$, invoking (3.18), by Taylor expansions and (3.21), one has

$$
\begin{aligned}
u^{0}\left(x_{\varepsilon}, t ; \nu\right)= & u_{0}^{0}\left(x_{\varepsilon}, t\right)+\nu u_{1}^{0}\left(x_{\varepsilon}, t\right)+\nu^{2} u_{2}^{0}\left(x_{\varepsilon}, t\right)+\cdots \\
= & u_{0}^{0}(x, t)+u_{0, x}^{0}(x, t) \varepsilon \psi+\frac{1}{2} u_{0, x x}^{0}(x, t)(\varepsilon \psi)^{2} \\
& +\nu\left(u_{1}^{0}(x, t)+u_{1, x}^{0}(x, t) \varepsilon \psi\right) \\
& +\nu^{2} u_{2}^{0}(x, t)+\cdots \\
= & u_{0}^{0}(x, t)+\nu\left(u_{0, x}^{0}(x, t) \sigma \psi+u_{1}^{0}(x, t)\right) \\
& +\nu^{2}\left(\frac{1}{2} u_{0, x x}^{0}(x, t)(\sigma \psi)^{2}+u_{1, x}^{0}(x, t) \sigma \psi+u_{2}^{0}(x, t)\right)+\cdots
\end{aligned}
$$

In a similar manner, we obtain, for $i=1,2$, that

$$
\begin{aligned}
u^{1}\left(x_{\varepsilon}, t ; \nu\right) & =u_{0}^{1}\left(x_{\varepsilon}, t\right)+\nu u_{1}^{1}\left(x_{\varepsilon}, t\right)+\cdots \\
& =u_{0}^{1}(x, t)+\nu\left(u_{0, x}^{1}(x, t) \sigma \psi+u_{1}^{1}(x, t)\right)+\cdots
\end{aligned}
$$

and

$$
u^{2}\left(x_{\varepsilon}, t ; \nu\right)=u_{0}^{2}\left(x_{\varepsilon}, t\right)+\cdots=u_{0}^{2}(x, t)+\cdots .
$$

Thus, (3.20) - (3.24) suggest us to define an ansatz for $u$ as follows

$$
u=u_{0}+\nu u_{1}+\nu^{2} u_{2}+\cdots,
$$

where, $u_{0}, u_{1}$ and $u_{2}$ are defined by

$$
\begin{aligned}
& u_{0}=u_{0}^{0}, \\
& u_{1}=u_{1}^{0}+\sigma u_{0}^{1}, \\
& u_{2}=u_{2}^{0}+\sigma u_{1}^{1}+\sigma^{2} u_{0}^{2} .
\end{aligned}
$$

Hence, there holds

$$
\begin{aligned}
u & =u_{0}+\nu\left(u_{1}+u_{0, x} \sigma \psi\right)+\nu^{2}\left(u_{2}+u_{1, x} \sigma \psi+\frac{1}{2} u_{0, x x}(\sigma \psi)^{2}\right)+\cdots \\
& =u_{0}+\nu I_{1}+\nu^{2} I_{2}+\cdots
\end{aligned}
$$

Here, $I_{1}, I_{2}$ denote

$$
I_{1}=u_{1}+u_{0, x} \sigma \psi, \quad I_{2}=u_{2}+u_{1, x} \sigma \psi+\frac{1}{2} u_{0, x x}(\sigma \psi)^{2} .
$$


The nonlinearity $F(u)$ can be expanded as

$$
\begin{aligned}
F(u)= & F\left(u_{0}\right)+\nu f\left(u_{0}\right)\left(u_{1}+u_{0, x} \sigma \psi\right)+\nu^{2} f\left(u_{0}\right)\left(u_{2}+u_{1, x} \sigma \psi+\frac{1}{2} u_{0, x x}(\sigma \psi)^{2}\right) \\
& +\nu^{2} \frac{1}{2} f^{\prime}\left(u_{0}\right)\left(u_{1}+u_{0, x} \sigma \psi\right)^{2}+\cdots \\
= & F\left(u_{0}\right)+\nu f\left(u_{0}\right) I_{1}+\nu^{2}\left(f\left(u_{0}\right) I_{2}+\frac{1}{2} f^{\prime}\left(u_{0}\right)\left(I_{1}\right)^{2}\right)+\cdots
\end{aligned}
$$

Inserting (3.29) into (3.1) and using (3.30) yield that (3.1) can be written in terms of $u^{i}$ and $\varepsilon$ as

$$
\begin{aligned}
0= & u_{t}+(F(u))_{x}-\nu u_{x x} \\
= & \left(u_{0}+\nu I_{1}+\nu^{2} I_{2}\right)_{t}-\nu\left(u_{0}+\nu I_{1}+\nu^{2} I_{2}\right)_{x x} \\
& +\left(F\left(u_{0}\right)\right)_{x}+\nu\left(f\left(u_{0}\right) I_{1}\right)_{x}+\nu^{2}\left(f\left(u_{0}\right) I_{2}+\frac{1}{2} f^{\prime}\left(u_{0}\right)\left(I_{1}\right)^{2}\right)_{x}+\cdots
\end{aligned}
$$

Then equating the coefficients of $\nu^{k}$ (where $\left.k=0,1,2\right)$ on both sides of equation (3.31), we thus deduce that

$$
\begin{array}{ll}
\nu^{0}: & \left(u_{0}\right)_{t}+\left(F\left(u_{0}\right)\right)_{x}=0, \\
\nu^{1}: & \left(u_{1}\right)_{t}+\left(f\left(u_{0}\right) u_{1}\right)_{x}=\mathcal{R}, \\
\nu^{2}: & \left(u_{2}\right)_{t}+\left(f\left(u_{0}\right) u_{2}\right)_{x}=\mathcal{R}_{1},
\end{array}
$$

where $\mathcal{R}$ and $\mathcal{R}_{1}$ are functions defined by

$$
\mathcal{R}:=u_{0, x x}-\sigma\left(u_{0, x} \psi^{\prime}+\left(\left(u_{0, x}\right)_{t}+\left(f\left(u_{0}\right) u_{0, x}\right)_{x}\right) \psi\right)
$$

and

$$
\begin{aligned}
\mathcal{R}_{1}:= & \left(u_{0, x} \sigma \psi+u_{1}\right)_{x x}-\left(\frac{1}{2} u_{0, x x}(\sigma \psi)^{2}+u_{1, x} \sigma \psi\right)_{t} \\
& -\left(f\left(u_{0}\right)\left(\frac{1}{2} u_{0, x x}(\sigma \psi)^{2}+u_{1, x} \sigma \psi\right)+\frac{1}{2} f^{\prime}\left(u_{0}\right)\left(u_{0, x} \sigma \psi+u_{1}\right)^{2}\right)_{x}
\end{aligned}
$$

from which we see that $\mathcal{R}, \mathcal{R}_{1}$ depend on the constant $\sigma$ and functions $\psi, u_{0}$ and $u_{1}$.

We now consider the expansion of the initial data. By using again Taylor expansions, (3.19) can be rewritten as

$$
\begin{aligned}
g^{\varepsilon}(x)= & g_{0}(x)+\sigma \nu\left(\psi(0) g_{0}^{\prime}(x)+g_{1}(x)\right) \\
& +\sigma^{2} \nu^{2}\left(\frac{1}{2} \psi(0)^{2} g_{0}^{\prime \prime}(x)+\psi(0) g_{1}^{\prime}(x)+g_{2}(x)\right)+\cdots .
\end{aligned}
$$

Thus this expansion suggests us to choose the initial data of $u_{i}(i=0,1,2)$ as follows

$$
\begin{aligned}
& \left.u_{0}\right|_{t=0}=\bar{g}_{0}, \\
& \left.u_{1}\right|_{t=0}=\bar{g}_{1}, \\
& \left.u_{2}\right|_{t=0}=\bar{g}_{2} .
\end{aligned}
$$


Here, $\bar{g}_{0}, \bar{g}_{1}, \bar{g}_{2}$ are defined by

$$
\begin{aligned}
& \bar{g}_{0}=g_{0} \\
& \bar{g}_{1}=\sigma\left(\psi(0) g_{0}^{\prime}+g_{1}\right), \\
& \bar{g}_{2}=\sigma^{2}\left(\frac{1}{2} \psi(0)^{2} g_{0}^{\prime \prime}+\psi(0) g_{1}^{\prime}+g_{2}\right) .
\end{aligned}
$$

From (3.41) it follows that $\nu \bar{g}_{1}$ is just the second term in (2.34). So the expansion of $g^{\varepsilon}$ obtained here coincides with the one carried out formally in (2.34) in Section 2.

To solve the equations (3.32)-(3.34) with initial data (3.37)-(3.39), we need to get the equations for $\varphi$ and $\psi$. So their solvability will be left to sub-Section 3.3, where the interface equations will be derived.

Part 2. Outer expansions of $v$. Now we turn to derive the outer expansions for $v$. It is not necessary to take into account again the effects of infinitesimal perturbations of the initial datum and infinitesimal translations of the shock position, since we have had, in hand, the equations for shock and its perturbation. We can construct directly an ansatz for $v$, that has the same form of (3.25), as follows

$$
v=v_{0}+\nu v_{1}+\nu^{2} v_{2}
$$

Similarly, from equation (3.3) we then have

$$
\begin{array}{ll}
\nu^{0}: & \left(v_{0}\right)_{t}+\left(f\left(u_{0}\right) v_{0}\right)_{x}=0, \\
\nu^{1}: & \left(v_{1}\right)_{t}+\left(f\left(u_{0}\right) v_{1}\right)_{x}=\delta_{1}, \\
\nu^{2}: & \left(v_{2}\right)_{t}+\left(f\left(u_{0}\right) v_{2}\right)_{x}=\delta_{2},
\end{array}
$$

and the initial data are

$$
\begin{aligned}
& \left.v_{0}\right|_{t=0}=h_{0}, \\
& \left.v_{1}\right|_{t=0}=h_{1}, \\
& \left.v_{2}\right|_{t=0}=h_{2} .
\end{aligned}
$$

Here $\delta_{1}, \delta_{2}$ are given by

$$
\begin{aligned}
& \delta_{1}=\left(v_{0}\right)_{x x}-\left(f^{\prime}\left(u_{0}\right) u_{1} v_{0}\right)_{x}, \\
& \delta_{2}=\left(v_{1}\right)_{x x}-\left(f^{\prime}\left(u_{0}\right) u_{2} v_{0}+\frac{1}{2} f^{\prime \prime}\left(u_{0}\right)\left(u_{1}\right)^{2} v_{0}\right)_{x} .
\end{aligned}
$$

By solving these problems (which are initial value problems of transport equations with discontinuous coefficients, for the well-posedness we refer to e.g. [6]), we then construct $v_{0}, v_{1}, v_{2}$ which are smooth up to the shock.

Part 3. Outer expansions of $p$. To get the outer expansions for $p$ we repeat again the procedure performed for $v$, similarly, from equation (3.7) we then have

$$
\begin{array}{ll}
\nu^{0}: & \left(p_{0}\right)_{s}-f\left(u_{0}\right)\left(p_{0}\right)_{x}=0, \\
\nu^{1}: & \left(p_{1}\right)_{s}-f\left(u_{0}\right)\left(p_{1}\right)_{x}=\beta_{1}, \\
\nu^{2}: & \left(p_{2}\right)_{s}-f\left(u_{0}\right)\left(p_{2}\right)_{x}=\beta_{2},
\end{array}
$$


and the initial data are

$$
\begin{aligned}
& \left.p_{0}\right|_{s=0}=p_{0}^{T}, \\
& \left.p_{1}\right|_{s=0}=p_{1}^{T}, \\
& \left.p_{2}\right|_{s=0}=p_{2}^{T},
\end{aligned}
$$

where $\beta_{1}, \beta_{2}$ are given by

$$
\begin{aligned}
& \beta_{1}=f^{\prime}\left(u_{0}\right) u_{1}\left(p_{0}\right)_{x}+\left(p_{0}\right)_{x x} \\
& \beta_{2}=f^{\prime}\left(u_{0}\right) u_{1}\left(p_{1}\right)_{x}+\left(f^{\prime}\left(u_{0}\right) u_{2}+\frac{f^{\prime \prime}\left(u_{0}\right)}{2}\left(u_{1}\right)^{2}\right)\left(p_{0}\right)_{x}+\left(p_{1}\right)_{x x} .
\end{aligned}
$$

Thus suppose $u_{0}, u_{1}, u_{2}$ are known (we will solve them in next sub-section), we are able to solve $p_{0}$, then insert $p_{0}$ into equations $p_{1}$, from the resulting linear equations we then can obtain $p_{1}$. Similarly, $p_{2}$ is obtained.

We are now going to discuss the solvability of problem (3.32), (3.37), problem (3.33), (3.38) and problem (3.34), (3.39). There are five unknowns, i.e. $u_{i}(i=0,1,2), \varphi$ and $\psi$, but only three equations. So, to form a complete system, as the first step, we need to find the equations that $\varphi$ and $\psi$ satisfy.

\subsection{Derivation of the interface equations}

This sub-section is devoted to deriving the interface equations from the first two terms of the outer expansion of $u$. This derivation is based upon an important observation that the values of outer expansions tend to those at the shock $\varphi(t)$, as the thickness $\nu$ of the quasi-shock region goes to zero. This is different from the usual way that one derives such equations from inner expansions, see, e.g. [15, 17]. One advantage of our approach

is that we can overcome the difficulty/limitation caused by the algebraically growth, as the fast variable $r_{\nu} \rightarrow \infty$ (the definition of $r_{\nu}$ is given in (3.70), $r_{\nu}$ is of order $O\left(\nu^{-1}\right)$ ), which is required by the matching conditions for the second term, i.e. $\tilde{u}_{1}$, of the inner expansion of $u$. One of the matching conditions is, for instance, (3.77) that reads

$$
\tilde{u}_{1}\left(r_{\nu}, t\right)=u_{1}(\varphi(t) \pm 0, t)+\left(r_{\nu}+\sigma \psi\right) \partial_{x} u_{0}(\varphi(t) \pm 0, t)+o(1),
$$

as $r_{\nu} \rightarrow \infty$. From which we see that $\tilde{u}_{1}\left(r_{\nu}, t\right)$ grows in $r_{\nu}$.

Therefore how to define the jump (e.g. $\tilde{u}_{1}(\infty, t)-\tilde{u}_{1}(-\infty, t)$, but $\tilde{u}_{1}( \pm \infty, t)$ may not exist) of the terms like $\tilde{u}_{1}$, of the inner expansion of $u$ ? We cannot define it in a usual way, some restrictions must be added, for instance, we assume that $\partial_{x} u_{0}(\varphi(t) \pm 0, t)=0$ for $\tilde{u}_{1}$, to ensure the values $\tilde{u}_{1}( \pm \infty, t)$ are finite, or we may define the jump of $\tilde{u}_{1}$ in a new manner: $\left[\tilde{u}_{1}\right](t)=\lim _{r \rightarrow \infty}\left(\tilde{u}_{1}(r, t)-\tilde{u}_{1}(-r, t)\right)$, so that $\left[\tilde{u}_{1}\right](t)$ in condition $(3.77)$ exists provided that $\partial_{x} u_{0}(\varphi(t)+0, t)=-\partial_{x} u_{0}(\varphi(t)-0, t)$ which, however, is still a restriction to $u_{0}$.

Assume that the limits of $u_{i}$ (or its derivatives) exist for any $t \in[0, T]$, from the left and right side of the shock, i.e.

$$
\partial_{x}^{k} u_{0}(\varphi(t) \pm 0, t), k=0,1, \text { and } u_{1}(\varphi(t) \pm 0, t) \text {, exist. }
$$


First of all, we derive the equation for the shock, and invoke equation (3.32) with initial datum (3.37) which is just a Cauchy problem for the unknown $u_{0}$. From the standard theory of conservation laws it then follows that

$$
\varphi^{\prime}\left[u_{0}\right]_{\varphi(t)}=\left[F\left(u_{0}\right)\right]_{\varphi(t)},
$$

which is the famous Rankine-Hugoniot condition.

Next we make use of (3.33) to find the equation for the variation of the shock position, i.e. $\psi$. We define, in a usual way, weak solution to problem (3.33) - (3.38), namely multiplying (3.33) by a test function $\zeta$ with compact support in $\mathbb{R} \times[0, T)$, and integrating it by parts. Then similar to the derivation of the Rankine-Hugoniot condition, we can find, as in e.g. Smoller [32], the equation of $\psi$ from this weak formulation by choosing a test function $\zeta$ with compact support contained in a small neighborhood $D=D_{1} \cup D_{2}$ of any fixed point $(x, t)$, see Figure 3 . Then applying integration by parts yields that

$$
\begin{aligned}
\sigma\left[u_{0}\right]_{\varphi(t)} \psi^{\prime}(t) & +\sigma\left(\left(-\varphi^{\prime}(t)\left[u_{0, x}\right]_{\varphi(t)}+\left[f\left(u_{0}\right) u_{0, x}\right]_{\varphi(t)}\right) \psi(t)\right) \\
& =\varphi^{\prime}(t)\left[u_{1}\right]_{\varphi(t)}-\left[f\left(u_{0}\right) u_{1}\right]_{\varphi(t)}+\left[u_{0, x}\right]_{\varphi(t)} .
\end{aligned}
$$

Here we used the fact that the integral vanishes over interior of $D_{1}$ and $D_{2}$, and that the values of $\zeta$ are equal to zero at the boundary of $D$.

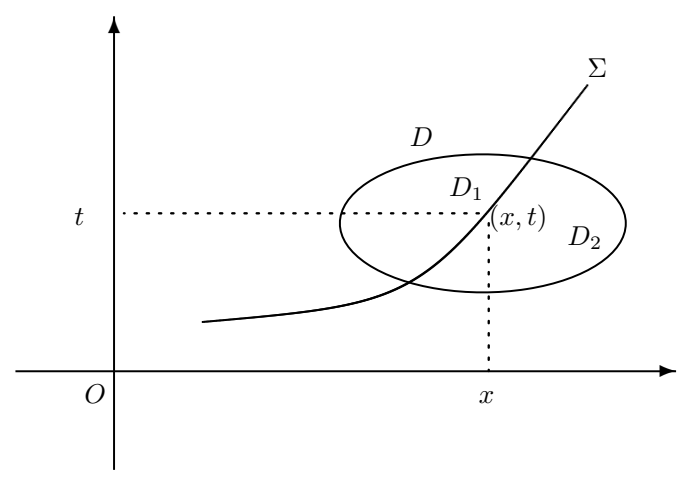

Figure 3: Subdomains $D_{1}$ and $D_{2}$.

Dividing equation (3.60) by $\sigma$ we obtain

$$
\begin{aligned}
{\left[u_{0}\right]_{\varphi(t)} \psi^{\prime}(t) } & +\left(-\varphi^{\prime}(t)\left[u_{0, x}\right]_{\varphi(t)}+\left[f\left(u_{0}\right) u_{0, x}\right]_{\varphi(t)}\right) \psi(t) \\
& =\frac{1}{\sigma}\left(\left(\varphi^{\prime}(t)\left[u_{1}\right]_{\varphi(t)}-\left[f\left(u_{0}\right) u_{1}\right]_{\varphi(t)}\right)+\left[u_{0, x}\right]_{\varphi(t)}\right) .
\end{aligned}
$$

Remark 3.1. By (3.27) we can rewrite the right-hand side of (3.61) as

$$
\begin{aligned}
& \frac{1}{\sigma}\left(\left(\varphi^{\prime}(t)\left[u_{1}\right]_{\varphi(t)}-\left[f\left(u_{0}\right) u_{1}\right]_{\varphi(t)}\right)+\left[u_{0, x}\right]_{\varphi(t)}\right) \\
= & \left(\varphi^{\prime}(t)\left[u_{0}^{1}\right]_{\varphi(t)}-\left[f\left(u_{0}\right) u_{0}^{1}\right]_{\varphi(t)}\right)+\frac{1}{\sigma}\left(\left(\varphi^{\prime}(t)\left[u_{1}^{0}\right]_{\varphi(t)}-\left[f\left(u_{0}\right) u_{1}^{0}\right]_{\varphi(t)}\right)+\left[u_{0, x}\right]_{\varphi(t)}\right) \\
\rightarrow & \varphi^{\prime}(t)\left[u_{0}^{1}\right]_{\varphi(t)}-\left[f\left(u_{0}\right) u_{0}^{1}\right]_{\varphi(t)} .
\end{aligned}
$$


Thus we see that if $f(u)=u$, equation (3.61) converges to the one derived in [8], i.e. equation (2.6) with $f(u)=u$.

We turn back to solvability of equation (3.61). To this end we need to determine a priori the quantity $\left[u_{1}\right]_{\varphi(t)}$. Invoking outer expansions of $u_{\nu}$ and $v_{\nu}$, noting that $v_{\nu}$ is the variation of $u_{\nu}$, we thus obtain two expansions of $u_{\nu}$, which are valid up to the shock,

$$
\begin{aligned}
u(x, t ; \nu, \sigma \nu)=: u_{\nu} & =u_{0}+\nu u_{1}+\cdots \\
& =u+\nu v_{\nu}+\cdots=u+\nu v_{0}+\cdots
\end{aligned}
$$

Here $u$ is chosen to be the unique entropy solution to problem (1.1)-(1.2). So it is natural to assume that $u_{0}=u$, whence $u_{1}=v_{0}$. Thus, there must hold

$$
\lim _{x \rightarrow \varphi(t)} u_{1}(x, t)=\lim _{x \rightarrow \varphi(t)} v_{0}(x, t)=v_{0}(\varphi(t), t)
$$

for any $t \in[0, T]$.

Therefore, we can write $\left[u_{1}\right]_{\varphi(t)}$ in terms of $\left[v_{0}\right]_{\varphi(t)}$. From $(3.59)$ we get $\varphi=\varphi(t)$ and insert it into (3.61). Hence one then solves (3.61) and gets $\psi$. Furthermore, we can solve $u_{0}$ from equation (3.32) with (3.37) which form a Riemann problem. Once $u_{0}$ is obtained, we see that the right hand side of (3.33) and (3.34), namely $\mathcal{R}, \mathcal{R}_{1}$ are also known quantities, thus problem (3.33) with (3.38) and problem (3.34) with (3.39) are linear in $u_{1}, u_{2}$ respectively, and can be solved easily to get $u_{1}, u_{2}$.

Combining the results obtained in sub-Sections 3.1 and 3.2, we then complete the construction of outer expansions for $u, v$ and $p$. We also find that the first term $v_{0}$ of the outer expansion of $v(x, t ; \nu, \varepsilon)$ can be expected to solve

$$
\begin{aligned}
\left(v_{0}\right)_{t}+\left(f\left(u_{0}\right) v_{0}\right)_{x} & =0, \text { in } Q^{+} \cup Q^{-} \\
{\left[u_{0}\right]_{\varphi(t)} \psi^{\prime}(t) } & +\left(-\varphi^{\prime}(t)\left[u_{0, x}\right]_{\varphi(t)}+\left[f\left(u_{0}\right) u_{0, x}\right]_{\varphi(t)}\right) \psi(t) \\
& =\frac{1}{\sigma}\left(\left(\varphi^{\prime}(t)\left[v_{0}\right]_{\varphi(t)}-\left[f\left(u_{0}\right) v_{0}\right]_{\varphi(t)}\right)+\left[u_{0, x}\right]_{\varphi(t)}\right), \\
\psi(0) & =\delta \varphi^{I} \\
v_{0}(x, 0) & =v^{I}(x), x \in\left\{x<\varphi^{I}\right\} \cup\left\{x>\varphi^{I}\right\} .
\end{aligned}
$$

Note that (2.6) is replaced by (3.66).

Remark 3.2. Solvability of $u_{1}^{0}$ and $u_{0}^{1}$. By the method of characteristics, we solve $u_{1}$ from problem (3.33), (3.38), and write it as

$$
u_{1}(x, t)=u_{1}(x(0), 0) \int_{0}^{t} \mathcal{R}(x, s) \cdot \exp \left(\int_{t}^{s}\left(f\left(u_{0}(x, \tau)\right)\right)_{x} d \tau\right) d s
$$

for $(x, t) \in Q^{+} \cup Q^{-}$, and $\psi$ from (3.61). Thus we see that $\psi, u_{1}$ depend on the parameter $\sigma$. On the other hand, under assumption that $\psi$ depends on $\sigma$, checking the construction we find that functions $u_{1}^{0}$ and $u_{0}^{1}$ are still independent of $\sigma$. Therefore, by formula (3.27) we assert that

$$
u_{1}^{0}=\left.u_{1}(x, t ; \sigma)\right|_{\sigma=0}=u_{1}(x(0), 0) \int_{0}^{t} u_{0, x x}(x, s) \exp \left(\int_{t}^{s}\left(f\left(u_{0}(x, \tau)\right)\right)_{x} d \tau\right) d s,
$$


whence

$$
u_{0}^{1}=\frac{1}{\sigma}\left(u_{1}-u_{1}^{0}\right) .
$$

Note that $u_{1}^{0}$ is the second term of outer expansions suppose that there is no perturbation of the initial datum. It is possible that the right hand side of (3.69) is independent of $\sigma$.

\subsection{Inner expansions}

An inner expansion is the one that is valid inside the quasi-shock region, which can be written as a series consisting of terms in the fast variable $r_{\nu}$ defined by

$$
r_{\nu}=\frac{d_{\nu}}{\nu}, \quad d_{\nu}=x-\varphi(t)-\sigma \nu \psi(t)
$$

(where $d_{\nu}$ is the so-called shifted slow variable), and in time $t$

$$
\tilde{\eta}=\tilde{\eta}\left(r_{\nu}, t, \nu\right)=\tilde{\eta}_{0}\left(r_{\nu}, t\right)+\nu \tilde{\eta}_{1}\left(r_{\nu}, t\right)+\nu^{2} \tilde{\eta}_{2}\left(r_{\nu}, t\right)+\cdots,
$$

here $\tilde{\eta}, \tilde{\eta}_{0}, \tilde{\eta}_{1}, \cdots$ will be replaced by $\tilde{u}, \tilde{u}_{0}, \tilde{u}_{1}, \cdots, \tilde{v}, \tilde{v}_{0}, \tilde{v}_{1}, \cdots$ and $\tilde{p}, \tilde{p}_{0}, \tilde{p}_{1}, \cdots$, respectively. In what follows, to simplify the notation, we use

$$
\tilde{h}^{\prime}=\frac{\partial}{\partial r_{\nu}} \tilde{h}
$$

for a function $\tilde{h}$ in fast variable and time $t$. Consequently

$$
\frac{\partial \tilde{h}}{\partial d_{\nu}}=\frac{1}{\nu} \tilde{h}^{\prime}, \quad \frac{\partial^{2} \tilde{h}}{\partial d_{\nu}^{2}}=\frac{1}{\nu^{2}} \tilde{h}^{\prime \prime} .
$$

We start the construction of inner expansions with the nonlinear terms

$$
\begin{aligned}
F(\tilde{\eta}) & =F\left(\tilde{\eta}_{0}\right)+\nu f\left(\tilde{\eta}_{0}\right) \tilde{\eta}_{1}+\nu^{2}\left(f\left(\tilde{\eta}_{0}\right) \tilde{\eta}_{2}+\frac{1}{2} f\left(\tilde{\eta}_{0}\right) \tilde{\eta}_{1}^{2}\right)+\cdots, \\
f(\tilde{\eta}) & =f\left(\tilde{\eta}_{0}\right)+\nu f^{\prime}\left(\tilde{\eta}_{0}\right) \tilde{\eta}_{1}+\nu^{2}\left(f^{\prime}\left(\tilde{\eta}_{0}\right) \tilde{\eta}_{2}+\frac{1}{2} f^{\prime \prime}\left(\tilde{\eta}_{0}\right) \tilde{\eta}_{1}^{2}\right)+\cdots .
\end{aligned}
$$

Therefore, inserting the ansatz into equation (3.1) of $u$ and equating both sides of the resulting equation yield

$$
\begin{aligned}
\nu^{-1}: & \tilde{u}_{0}^{\prime \prime}+\dot{\varphi} \tilde{u}_{0}^{\prime}-\left(F\left(\tilde{u}_{0}\right)\right)^{\prime}=0, \\
\nu^{0}: & \tilde{u}_{1}^{\prime \prime}+\dot{\varphi} \tilde{u}_{1}^{\prime}-\left(f\left(\tilde{u}_{0}\right) \tilde{u}_{1}\right)^{\prime}=-\sigma \dot{\psi} \tilde{u}_{0}^{\prime}+\tilde{u}_{0, t}, \\
\nu^{1}: & \tilde{u}_{2}^{\prime \prime}+\dot{\varphi} \tilde{u}_{2}^{\prime}-\left(f\left(\tilde{u}_{0}\right) \tilde{u}_{2}\right)^{\prime}=-\sigma \dot{\psi} \tilde{u}_{1}^{\prime}+\left(\frac{f^{\prime}\left(\tilde{u}_{0}\right)}{2} \tilde{u}_{1}^{2}\right)^{\prime}+\tilde{u}_{1, t} .
\end{aligned}
$$

These are ordinary differential equations of second order, thus two conditions are needed for each equation to ensure the uniqueness. Such conditions can be obtained from matching conditions, which make the inner and outer expansions coincide asymptotically in an intermediate region, say $\mathcal{M}$. Over this region, there holds

$$
\tilde{u}_{0}\left(r_{\nu}, t\right)+\nu \tilde{u}_{1}\left(r_{\nu}, t\right)+\nu^{2} \tilde{u}_{2}\left(r_{\nu}, t\right)=u_{0}(x, t)+\nu u_{1}(x, t)+\nu^{2} u_{2}(x, t)+O\left(\nu^{3}\right), \text { in } \mathcal{M},
$$


as $r_{\nu} \rightarrow \pm \infty$ and $x$ is close to the shock $\varphi(t)$.

By definition, we can rewrite $x=\nu\left(r_{\nu}+\sigma \psi(t)\right)+\varphi(t)$. Using Taylor expansions one thus can obtain the matching conditions in the following form

$$
\begin{aligned}
\tilde{u}_{0}\left(r_{\nu}, t\right)= & u_{0}(\varphi(t) \pm 0, t)+o(1) \\
\tilde{u}_{1}\left(r_{\nu}, t\right)= & u_{1}(\varphi(t) \pm 0, t)+\left(r_{\nu}+\sigma \psi\right) \partial_{x} u_{0}(\varphi(t) \pm 0, t)+o(1), \\
\tilde{u}_{2}\left(r_{\nu}, t\right)= & u_{2}(\varphi(t) \pm 0, t)+\left(r_{\nu}+\sigma \psi\right) \partial_{x} u_{1}(\varphi(t) \pm 0, t) \\
& +\frac{\left(r_{\nu}+\sigma \psi\right)^{2}}{2} \partial_{x}^{2} u_{0}(\varphi(t) \pm 0, t)+o(1),
\end{aligned}
$$

as $r_{\nu} \rightarrow \pm \infty$. For more details, we refer, e.g. to the book by Fife [15]. Later on we will prove $o(1)=\exp \left(-c\left|r_{\nu}\right|\right)$ in all the matching conditions, where $c$ is a fixed positive number.

Next we are going to solve the problems consisting of $(3.73)-(3.75)$ and the corresponding boundary conditions (3.76) - (3.78). We firstly solve (3.73). To this end, we study the solvability of the following problem

$$
\begin{aligned}
& \hat{\phi}^{\prime \prime}+\sigma \hat{\phi}^{\prime}-F(\hat{\phi})^{\prime}=0, \\
& \hat{\phi}(\xi) \rightarrow u_{ \pm}, \text {as } \xi \rightarrow \pm \infty,
\end{aligned}
$$

since the solution $\hat{\phi}=\hat{\phi}(\xi)$ of this problem is closely related to that of (3.73), as we shall see later. Here $\sigma$ satisfies

$$
\sigma\left(u_{-}-u_{+}\right)=F\left(u_{-}\right)-F\left(u_{+}\right)
$$

From this, integrating equation (3.79) yields

$$
\hat{\phi}^{\prime}=F(\hat{\phi})-F\left(u_{-}\right)-\sigma\left(\hat{\phi}-u_{-}\right) .
$$

For the solution $\hat{\phi}=\hat{\phi}\left(\xi ; u_{-}, \sigma\right)$, the following estimates are obtained (see also [21]), as $\xi \rightarrow-\infty$,

$$
\begin{aligned}
\hat{\phi}\left(\xi ; u_{-}, \sigma\right)-u_{-} & =O(1)\left|u_{+}-u_{-}\right| \mathrm{e}^{-c|\xi|} \\
\frac{\partial \hat{\phi}}{\partial u_{-}}-1 & =O(1) \mathrm{e}^{-c|\xi|} \\
\frac{\partial \hat{\phi}}{\partial \sigma} & =O(1) \mathrm{e}^{-c|\xi|}
\end{aligned}
$$

Similarly, as $\xi \rightarrow+\infty$, we have

$$
\begin{aligned}
\hat{\phi}\left(\xi ; u_{-}, \sigma\right)-u_{+} & =O(1)\left|u_{+}-u_{-}\right| \mathrm{e}^{-c|\xi|} \\
\frac{\partial \hat{\phi}}{\partial u_{-}}-\frac{u_{+}}{u_{-}} & =O(1) \mathrm{e}^{-c|\xi|} \\
\frac{\partial \hat{\phi}}{\partial \sigma}-\frac{\partial u_{+}}{\partial \sigma} & =O(1) \mathrm{e}^{-c|\xi|}
\end{aligned}
$$

Define

$$
\sigma=\dot{\varphi}(t), u_{ \pm}=u(\varphi(t) \pm 0, t), \xi=r_{\nu}
$$


and $\tilde{u}_{0}=\hat{\phi}\left(\xi ; u_{-}, \sigma\right)$.

Then $\tilde{u}_{0}$ is a solution to problem (3.73), (3.76).

Now we construct $\tilde{u}_{1}$. From matching condition $(3.77)$ we can expect that $\tilde{u}_{1}\left(r_{\nu}, t\right)$ has the following form

$$
\tilde{u}_{1}\left(r_{\nu}, t\right)=\tilde{u}_{1 a}\left(r_{\nu}, t\right)+\tilde{u}_{1 b}\left(r_{\nu}, t\right),
$$

here $\tilde{u}_{1 b}\left(r_{\nu}, t\right)$ is a smooth function such that

$$
\tilde{u}_{1 b}\left(r_{\nu}, t\right)= \begin{cases}r_{\nu} \partial_{x} u_{0}(\varphi(t)-0, t), & \text { if } r_{\nu}<-A \\ r_{\nu} \partial_{x} u_{0}(\varphi(t)+0, t), & \text { if } r_{\nu}>A\end{cases}
$$

for a sufficiently large positive number $A$. Using the decay estimates for $\tilde{u}_{0}\left(r_{\nu}, t\right)$ and the identity

$$
\frac{d}{d t} u_{0}(\varphi(t) \pm 0, t)=\left(\dot{\varphi}(t)-f\left(u_{0}(\varphi(t) \pm 0, t)\right)\right) u_{0 x}(\varphi(t) \pm 0, t)
$$

one then arrives at

$$
\tilde{u}_{1 a}^{\prime \prime}+\dot{\varphi} \tilde{u}_{1 a}^{\prime}-\left(f\left(\tilde{u}_{0}\right) \tilde{u}_{1 a}\right)^{\prime}=-\sigma \dot{\psi} \tilde{u}_{0}^{\prime}+e\left(r_{\nu}, t\right)
$$

here $e=e\left(r_{\nu}, t\right)$ is an error satisfying that for large $\left|r_{\nu}\right|$,

$$
\left|e\left(r_{\nu}, t\right)\right| \leq C \exp \left(-c\left|r_{\nu}\right|\right)
$$

Therefore, equation (3.74) is reduced to

$$
\tilde{u}_{1 a}^{\prime}+\left(\dot{\varphi}-f\left(\tilde{u}_{0}\right)\right) \tilde{u}_{1 a}=-\sigma \dot{\psi} \tilde{u}_{0}+E\left(r_{\nu}, t\right)+C(t)
$$

with $C(t)$ being an arbitrary integral constant depending only on $t$ and $E=E\left(r_{\nu}, t\right)$ is defined by

$$
E=\int_{0}^{r_{\nu}} e(\xi, t) d \xi
$$

Invoking that we have solved $\psi$, we see that the right hand side of (3.89) is known. Thus from this equation we obtain $\tilde{u}_{1 a}$, hence $\tilde{u}_{1}$ too. By choosing suitable $C(t)$, we obtain that $\tilde{u}_{1}$ satisfies the matching condition in (3.77) with $o(1)=e^{-c\left|r_{\nu}\right|}$.

In a similar way, $\tilde{u}_{2}$ can be constructed and the details are omitted here. We thus obtain $\tilde{u}_{0}, \tilde{u}_{1}, \tilde{u}_{2}$, and the construction is finished too.

Construction of inner expansions for $v$. Straightforward computations yield the equations for the inner expansion of $v$

$$
\begin{array}{cl}
\nu^{-1}: & \tilde{v}_{0}^{\prime \prime}+\dot{\varphi} \tilde{v}_{0}^{\prime}-\left(f\left(\tilde{u}_{0}\right) \tilde{v}_{0}\right)^{\prime}=0, \\
\nu^{0}: & \tilde{v}_{1}^{\prime \prime}+\dot{\varphi} \tilde{v}_{1}^{\prime}-\left(f\left(\tilde{u}_{0}\right) \tilde{v}_{1}\right)^{\prime}=f_{1}, \\
\nu^{1}: & \tilde{v}_{2}^{\prime \prime}+\dot{\varphi} \tilde{v}_{2}^{\prime}-\left(f\left(\tilde{u}_{0}\right) \tilde{v}_{2}\right)^{\prime}=f_{2},
\end{array}
$$

where $f_{1}$ and $f_{2}$ are defined by

$$
\begin{aligned}
& f_{1}=\tilde{v}_{0, t}-\sigma \dot{\psi} \tilde{v}_{0}^{\prime}+\left(f^{\prime}\left(\tilde{u}_{0}\right) \tilde{u}_{1} \tilde{v}_{0}\right)^{\prime} \\
& f_{2}=\tilde{v}_{1, t}-\sigma \dot{\psi} \tilde{v}_{1}^{\prime}+\left(f^{\prime}\left(\tilde{u}_{0}\right) \tilde{u}_{1} \tilde{v}_{1}+\left(f^{\prime}\left(\tilde{u}_{0}\right) \tilde{u}_{2}+\frac{1}{2} f^{\prime \prime}\left(\tilde{u}_{0}\right) \tilde{u}_{1}^{2}\right) \tilde{v}_{0}\right)^{\prime} .
\end{aligned}
$$


Using the matching conditions

$$
\begin{aligned}
\tilde{v}_{0}\left(r_{\nu}, t\right)= & v_{0}(\varphi(t) \pm 0, t)+o(1) \\
\tilde{v}_{1}\left(r_{\nu}, t\right)= & v_{1}(\varphi(t) \pm 0, t)+\left(r_{\nu}+\sigma \psi\right) \partial_{x} v_{0}(\varphi(t) \pm 0, t)+o(1), \\
\tilde{v}_{2}\left(r_{\nu}, t\right)= & v_{2}(\varphi(t) \pm 0, t)+\left(r_{\nu}+\sigma \psi\right) \partial_{x} v_{1}(\varphi(t) \pm 0, t) \\
& +\frac{\left(r_{\nu}+\sigma \psi\right)^{2}}{2} \partial_{x}^{2} v_{0}(\varphi(t) \pm 0, t)+o(1)
\end{aligned}
$$

solving problems (3.90) - (3.92) and boundary conditions (3.95) - (3.97), we then obtain successively $\tilde{v}_{0}, \tilde{v}_{1}, \tilde{v}_{2}$ by the constructive proof similar to that for $\tilde{v}_{1}$.

\section{Construction of inner expansions for $p$.}

Finally we consider the inner expansion of $p$, where an interesting phenomenon arises: the approximate solution of $p$ approximates to a constant in the region occupied by the set of characteristics which form the shock.

The first three terms of the inner expansion of $p$ can be obtained by solving the following equations

$$
\begin{array}{cl}
\nu^{-1}: & \tilde{p}_{0}^{\prime \prime}-\left(\dot{\varphi}-f\left(\tilde{u}_{0}\right)\right) \tilde{p}_{0}^{\prime}=0, \\
\nu^{0}: & \tilde{p}_{1}^{\prime \prime}-\left(\dot{\varphi}-f\left(\tilde{u}_{0}\right)\right) \tilde{p}_{1}^{\prime}=f_{3}, \\
\nu^{1}: & \tilde{p}_{2}^{\prime \prime}-\left(\dot{\varphi}-f\left(\tilde{u}_{0}\right)\right) \tilde{p}_{2}^{\prime}=f_{4},
\end{array}
$$

where $f_{3}$ and $f_{4}$ are defined by

$$
\begin{aligned}
& f_{3}=\tilde{p}_{0, s}+\left(f^{\prime}\left(\tilde{u}_{0}\right) \tilde{u}_{1}+\sigma \dot{\psi}\right) \tilde{p}_{0}^{\prime}, \\
& f_{4}=\tilde{p}_{1, s}+f^{\prime}\left(\tilde{u}_{0}\right) \tilde{u}_{1} \tilde{p}_{1}+\left(f^{\prime}\left(\tilde{u}_{0}\right) \tilde{u}_{2}+\frac{1}{2} f^{\prime \prime}\left(\tilde{u}_{0}\right) \tilde{u}_{1}^{2}\right) \tilde{p}_{0}^{\prime}+\sigma \dot{\psi} \tilde{p}_{1}^{\prime} .
\end{aligned}
$$

And the following matching conditions should be satisfied, as $r_{\nu} \rightarrow \pm \infty$,

$$
\begin{aligned}
\tilde{p}_{0}\left(r_{\nu}, t\right)= & p_{0}(\varphi(t) \pm 0, t)+o(1) \\
\tilde{p}_{1}\left(r_{\nu}, t\right)= & p_{1}(\varphi(t) \pm 0, t)+\left(r_{\nu}-\sigma \psi\right) \partial_{x} p_{0}(\varphi(t) \pm 0, t)+o(1), \\
\tilde{p}_{2}\left(r_{\nu}, t\right)= & p_{2}(\varphi(t) \pm 0, t)+\left(r_{\nu}-\sigma \psi\right) \partial_{x} p_{1}(\varphi(t) \pm 0, t) \\
& +\frac{\left(r_{\nu}-\sigma \psi\right)^{2}}{2} \partial_{x}^{2} p_{0}(\varphi(t) \pm 0, t)+o(1) .
\end{aligned}
$$

Now, we show the existence of $\tilde{p}_{0}, \tilde{p}_{1}$ and $\tilde{p}_{2}$. Integrating (3.98) with respect to $r_{\nu}$, we have

$$
\tilde{p}_{0}^{\prime}\left(r_{\nu}, T-s\right)=C \exp \left\{\int_{0}^{r_{\nu}}\left[\dot{\varphi}-f\left(\tilde{u}_{0}\right)\right] d \eta\right\}+C(s) .
$$

In view of the entropy condition, we infer that $\tilde{p}_{0}^{\prime} \rightarrow \infty$ as $r_{\nu} \rightarrow \infty$ unless $\tilde{p}_{0}^{\prime} \equiv 0$. Therefore,

$$
\tilde{p}_{0}\left(r_{\nu}, s\right) \equiv C(s), \quad \forall r_{\nu} \in(-\infty,+\infty) .
$$

We are in a position to show that $\tilde{p}_{0} \equiv$ constant.

Suppose that $s_{1} \in[0, T]$. For any given constant $G>0$, let $A=\left(x_{1}, T-s_{1}\right)$ (see Figure 4 ) be any point in the region where the inner expansion is valid, to the left hand 


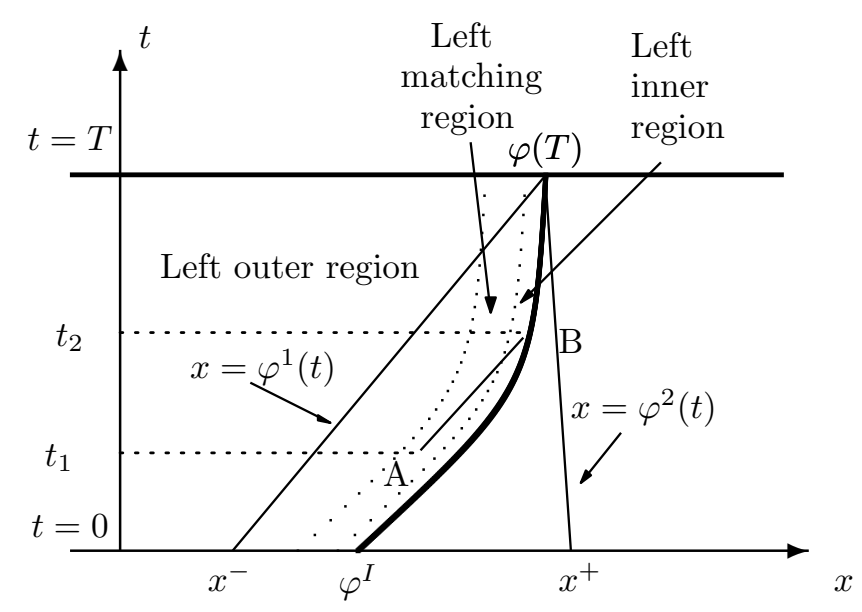

Figure 4: Solving the leading order solutions to the dual problem (to the left side of the shock).

side of the shock for instance, such that $\xi_{1}=\nu^{-1}\left(x_{1}-\varphi\left(T-s_{1}\right)\right)+\sigma \psi\left(T-s_{1}\right)$ with $\left|\xi_{1}\right|>G$.

Then by (3.107) and the matching condition, we have

$$
\tilde{p}_{0}\left(\xi_{1}, T-s_{1}\right)=C\left(s_{1}\right)
$$

and

$$
p_{0}\left(x_{1}, T-s_{1}\right)=C\left(s_{1}\right)+O(1) e^{-\alpha\left|\xi_{1}\right|} .
$$

Moreover, we assume that the characteristic line crossing $A$ intersects the shock at $B=$ $\left(\varphi\left(T-s_{2}\right), T-s_{2}\right)$. Then, by the method of characteristics, we obtain that

$$
p_{0}\left(\varphi\left(T-s_{2}\right)-0, s_{2}\right)=C\left(s_{1}\right)+O(1) e^{-\alpha\left|\xi_{1}\right|} .
$$

From the matching condition and (3.107), we reach

$$
\tilde{p}_{0}\left(\xi_{1}, s_{2}\right)=C\left(s_{2}\right)=C\left(s_{1}\right)+O(1) e^{-\alpha\left|\xi_{1}\right|} .
$$

Since $s_{1}, \xi_{1}$ (or $s_{2}$ ) can be chosen to be arbitrary, we conclude by letting $\xi_{1} \rightarrow \infty$ that $\tilde{p}_{0}\left(r_{\nu}, s\right) \equiv C_{0}$ for all $r_{\nu} \in(-\infty,+\infty)$ and $s \in[0, T]$, and $p_{0}(x, s)=C_{0}$ in the region occupied by the set of characteristics forming the shock $x=\varphi(T-s)$.

Similarly, we can show that $\tilde{p}_{1}=p_{1}=$ constant. Moreover, we obtain the existence of $\tilde{p}_{2}$ and $p_{2}$ from

$$
\begin{aligned}
& p_{2, s}-f\left(u_{0}(x, t)\right) p_{2, x}=0, \\
& \tilde{p}_{2}^{\prime \prime}-\left[\dot{\varphi}-f\left(\bar{u}_{0}\right)\right] \tilde{p}_{2}^{\prime}=f\left(\bar{u}_{0}\right) \bar{u}_{1} \tilde{p}_{1}, \\
& \tilde{p}_{2}( \pm \infty, s)=p_{2}(\varphi(T-s) \pm 0, s) .
\end{aligned}
$$

Note that $p_{2}, \tilde{p}_{2}=O(\zeta)$ as $r_{\nu} \rightarrow \infty$, however, $\nu p_{2}, \nu \tilde{p}_{2} \rightarrow 0$ as $\nu \rightarrow 0$. Indeed, the growth speed for the inner approximate solutions to $p$ are lower than those of $u$ and $v$. It results in that the corresponding convergent rate should be not worse than the ones for the conservation law and its linearized equation. 
Remark 3.3. For the terminal data $p^{T}(x)$, which is continuous at $(\varphi(T), T)$, the remained work is to construct the approximate solution from these inner and outer expansions, and to show the convergence of approximate solution. However, for the piecewise smooth terminal data $p^{T}(x)$ with a discontinuity at $(\varphi(T), T)$, some modifications should be done. We still need to expand the solution along two characteristics $x=\varphi^{1}(t)$ and $x=\varphi^{2}(t)$, since there are discontinuities there for the solution to the inviscid dual problem. Here $x=\varphi^{1}(t)$ and $x=\varphi^{2}(t)$ are the characteristics across $(\varphi(T), T)$, which are lying on the left hand side and right hand side of the shock $x=\varphi(t)$, respectively. It is not hard to obtain the new inner and outer expansions for $p$, with Riemann-type terminal data for instance, along these two characteristics.

Therefore, we have constructed the inner and outer expansions up to $\nu^{2}$, for $u, v, p$ respectively, which can be written as follows

$$
\begin{aligned}
U_{2}(x, t) & =\left(u_{0}+\nu u_{1}+\nu^{2} u_{2}\right)(x, t), \\
\tilde{U}_{2}\left(r_{\nu}, t\right) & =\left(\tilde{u}_{0}+\nu \tilde{u}_{1}+\nu^{2} \tilde{u}_{2}\right)\left(r_{\nu}, t\right) . \\
V_{2}(x, t) & =\left(v_{0}+\nu v_{1}+\nu^{2} v_{2}\right)(x, t), \\
\tilde{V}_{2}\left(r_{\nu}, t\right) & =\left(\tilde{v}_{0}+\nu \tilde{v}_{1}+\nu^{2} \tilde{v}_{2}\right)\left(r_{\nu}, t\right) . \\
P_{2}(x, t) & =\left(p_{0}+\nu p_{1}+\nu^{2} p_{2}\right)(x, t), \\
\tilde{P}_{2}\left(r_{\nu}, t\right) & =\left(\tilde{p}_{0}+\nu \tilde{p}_{1}+\nu^{2} \tilde{p}_{2}\right)\left(r_{\nu}, t\right) .
\end{aligned}
$$

\subsection{Approximate solutions}

In this sub-section we shall make use of a suitable cut-off function to combine together the outer and inner expansions constructed in sub-Sections 3.1, 3.3 and 3.4, whence the approximate solutions will be obtained.

Define a function $\chi=\chi(\xi): \mathbb{R} \rightarrow \mathbb{R}^{+}$which is smooth such that

$$
\chi(\xi)= \begin{cases}1 & \text { if }|\xi| \leq 1 \\ 0 & \text { if }|\xi| \geq 2\end{cases}
$$

and $0 \leq \chi(\xi) \leq 1$ if $|\xi| \in[1,2]$.
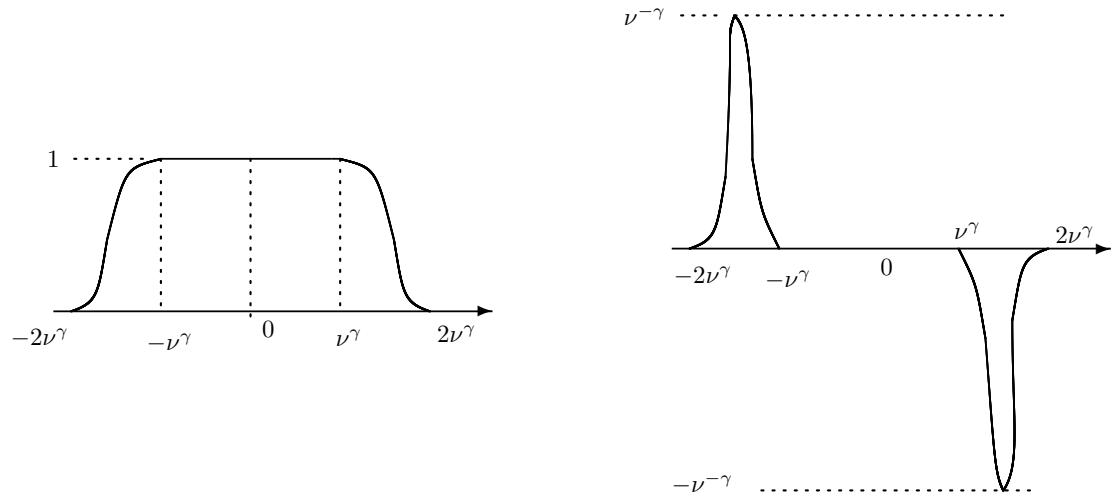

Figure 5: Typical shapes of functions $\chi_{\nu}$ and $\chi_{\nu}^{\prime}$. 
For fixed $t$, let

$$
\chi_{\nu}(x, t)=\chi\left(\nu^{1-\gamma} \frac{x-\varphi(t)}{\nu}\right)=\chi\left(\nu^{-\gamma}(x-\varphi(t))\right),
$$

which is easily seen from definition that

$$
\operatorname{supp}\left(\chi_{\nu}\right) \subset\left[-2 \nu^{\gamma}, 2 \nu^{\gamma}\right], \quad \operatorname{supp}\left(\chi_{\nu}^{\prime}\right), \operatorname{supp}\left(\chi_{\nu}^{\prime \prime}\right) \subset\left[\nu^{\gamma}, 2 \nu^{\gamma}\right] \cup\left[-2 \nu^{\gamma},-\nu^{\gamma}\right] .
$$

Here $\gamma \in(0,1)$ whose range will be decided later.

From the expansions $(3.111)$ - (3.112), (3.113) - (3.114) and (3.115) - (3.116), we are in a position to construct, respectively, the approximate solutions $\hat{U}_{2}^{\nu}, \hat{V}_{2}^{\nu}$ and $\hat{P}_{2}^{\nu}$ as follows

$$
\begin{aligned}
\hat{U}_{2}^{\nu}(x, t) & =\chi_{\nu}(x, t) \tilde{U}_{2}\left(r_{\nu}, t\right)+\left(1-\chi_{\nu}(x, t)\right) U_{2}(x, t), \\
\hat{V}_{2}^{\nu}(x, t) & =\chi_{\nu}(x, t) \tilde{V}_{2}\left(r_{\nu}, t\right)+\left(1-\chi_{\nu}(x, t)\right) V_{2}(x, t), \\
\hat{P}_{2}^{\nu}(x, t) & =\chi_{\nu}(x, t) \tilde{P}_{2}\left(r_{\nu}, t\right)+\left(1-\chi_{\nu}(x, t)\right) P_{2}(x, t) .
\end{aligned}
$$

By definition, if $(x, t)$ is sufficiently close to the quasi-shock region, then $\chi_{\nu}(x, t)=1$, thus the approximate solution $\hat{U}_{2}^{\nu}$ is equal to the inner expansion $\tilde{U}_{2}$, namely

$$
\hat{U}_{2}^{\nu}(x, t)=\tilde{U}_{2}\left(r_{\nu}, t\right) .
$$

On the other hand, suppose that if $(x, t)$ is sufficiently away from the quasi-shock region, then $1-\chi_{\nu}(x, t)=1$ which yields that the approximate solution is just the outer expansion, i.e.

$$
\hat{U}_{2}^{\nu}(x, t)=U_{2}(x, t) .
$$

In the intermediate region, there hold $0 \leq \chi_{\nu}(x, t) \leq 1$ and $\tilde{U}_{2}\left(r_{\nu}, t\right), U_{2}(x, t)$ are equal asymptotically which can be seen from the matching conditions, thus we can replace one of the two expansions by one another with a small error, to be precise, we write

$$
\hat{U}_{2}^{\nu}(x, t)=\chi_{\nu}(x, t) \tilde{U}_{2}\left(r_{\nu}, t\right)+\left(1-\chi_{\nu}(x, t)\right) \tilde{U}_{2}\left(r_{\nu}, t\right)+o(1)=\tilde{U}_{2}\left(r_{\nu}, t\right)+o(1),
$$

also there holds $\hat{U}_{2}^{\nu}(x, t)=U_{2}(x, t)+o(1)$. Therefore, the approximate solution is a good combination of inner and outer expansions.

In what follows we will omit arguments $(x, t),\left(r_{\nu}, t\right)$ and so on, for simplicity. Our first convergence result is that the approximate solutions $\hat{U}_{2}^{\nu}, \hat{V}_{2}^{\nu}, \hat{P}_{2}^{\nu}$ satisfy asymptotically their corresponding equation.

Theorem 3.1 Suppose that the assumptions (3.9) - (3.11) are satisfied and that $\varepsilon=\sigma \nu$ with $\sigma$ being a positive constant.

Then the approximate solutions $\hat{U}_{2}^{\nu}, \hat{V}_{2}^{\nu}, \hat{P}_{2}^{\nu}$ satisfy respectively, equations (3.1), (3.3) and (3.5), in the following sense

$$
\begin{aligned}
\left(\hat{U}_{2}^{\nu}\right)_{t}-\nu\left(\hat{U}_{2}^{\nu}\right)_{x x}+\left(F\left(\hat{U}_{2}^{\nu}\right)\right)_{x} & =O\left(\nu^{\alpha}\right), \\
\left(\hat{V}_{2}^{\nu}\right)_{t}-\nu\left(\hat{V}_{2}^{\nu}\right)_{x x}+\left(f\left(\hat{U}_{2}^{\nu}\right) \hat{V}_{2}^{\nu}\right)_{x} & =O\left(\nu^{\alpha}\right), \\
-\left(\hat{P}_{2}^{\nu}\right)_{t}-\nu\left(\hat{P}_{2}^{\nu}\right)_{x x}-f\left(\hat{U}_{2}^{\nu}\right)\left(\hat{P}_{2}^{\nu}\right)_{x} & =O\left(\nu^{\alpha}\right),
\end{aligned}
$$

where $\alpha=3 \gamma-1$ and $\gamma \in\left(\frac{2}{3}, 1\right)$.

We leave the proof of Theorem 3.1 to the next section. 


\section{Convergence of the approximate solutions}

This section is concerned with the proof of following theorem, i.e. Theorem 4.1, which consists of two parts, one is to prove Theorem 3.1 which asserts that the equations are satisfied asymptotically, and another is to investigate the convergence rate (with respect to the small parameter $\nu$ ), by employing the method of energy estimates and the construction of approximate solutions.

Theorem 4.1 Suppose that the assumptions in Theorem 3.1 are met. Let u,p be, respectively, the unique entropy solution with only one shock, the reversible solution to problems (1.1) - (1.2) and (1.8) - (1.9), and let $v$ be the unique solutions to problem (3.65) - (3.68), such that $\max _{x \in[-R, R]} p^{T}(x) \leq C$ for some constant $R>0$ and

$$
\int_{0}^{T} \int_{\{x \neq \varphi(t), t \in[0, T]\}} \sum_{i=1}^{6}\left(\left|\partial_{x}^{i} u(x, t)\right|^{2}+\left|\partial_{x}^{i} v(x, t)\right|^{2}+\left|\partial_{x}^{i} p(x, t)\right|^{2}\right) d x d t \leq C .
$$

Then the solutions $\left(u^{\nu}, v^{\nu}, p^{\nu}\right)$ of problems (3.1) - (3.2), (3.3) - (3.4) and (3.5) (3.6) converge, respectively, to $(u, v, p)$ in $\left[L^{\infty}\left(0, T ; L^{2}(\mathbb{R})\right)\right]^{3}$ as $\nu \rightarrow 0$, and the following estimates hold

$$
\sup _{0 \leq t \leq T}\left(\left\|u^{\nu}(t)-u(t)\right\|+\left\|v^{\nu}(t)-v(t)\right\|+\left\|p^{\nu}(t)-p(t)\right\|\right) \leq C_{\eta} \nu^{\eta} .
$$

Moreover, we also have in a sub-domain the following estimate

$$
\sup _{0 \leq t \leq T}\left\|p^{\nu}-p\right\|_{L^{\infty}\left(\Omega_{h}\right)} \leq C_{h} \nu \rightarrow 0
$$

as $\nu \rightarrow 0$. Here $\eta$ is a constant defined by

$$
\eta=\frac{1+\gamma}{2} \text {, where } \gamma \text { is the same as in Theorem 3.1, hence } \eta \in\left(\frac{5}{6}, 1\right) \text {, }
$$

and $C_{\eta}$ (res. $C_{h}$ ) denotes a constant depending only on parameters $\eta$ (res. $h$ ). The domain $\Omega_{h}$ is defined, for any positive constant $h$, by

$$
\Omega_{h}=\left\{(x, t) \in Q_{T}|| x-\varphi(t) \mid>h\right\} .
$$

Remark 4.1. These convergence results ensure us to select alternating descent directions for optimal control problem of viscous scalar conservation laws in a similar manner as done for inviscid ones in [11].

\subsection{The equations are satisfied asymptotically}

In this sub-section, we are going to prove that the approximate solutions $\hat{U}_{2}^{\nu}, \hat{V}_{2}^{\nu}, \hat{P}_{2}^{\nu}$ satisfy asymptotically, the corresponding equations (3.1), (3.3) and (3.7) respectively. We omit, in this sub-section, the superscript $\nu$ and write the approximate solutions as $\hat{U}_{2}, \hat{V}_{2}, \hat{P}_{2}$, to simplify notations.

Proof of Theorem 3.1. We divide the proof into three parts. 
Part 1. Firstly we investigate the convergence of $\hat{U}_{2}$. Here and hereafter, in order to simplify notations, we introduce a new variable $d=d(x, t)$ defined by

$$
d(x, t)=x-\varphi(t) .
$$

Straightforward computations yield

$$
\begin{aligned}
\left(\hat{U}_{2}\right)_{t}= & \frac{d_{t}}{\nu^{\gamma}} \chi_{\nu}^{\prime}\left(\tilde{U}_{2}-U_{2}\right)+\chi_{\nu} \cdot\left(\tilde{U}_{2}\right)_{t}+\left(1-\chi_{\nu}\right)\left(U_{2}\right)_{t} \\
\left(\hat{U}_{2}\right)_{x}= & \frac{1}{\nu^{\gamma}} \chi_{\nu}^{\prime}\left(\tilde{U}_{2}-U_{2}\right)+\chi_{\nu} \cdot\left(\tilde{U}_{2}\right)_{x}+\left(1-\chi_{\nu}\right)\left(U_{2}\right)_{x} \\
\left(\hat{U}_{2}\right)_{x x}= & \frac{1}{\nu^{2 \gamma}} \chi_{\nu}^{\prime \prime}\left(\tilde{U}_{2}-U_{2}\right)+\frac{2}{\nu^{\gamma}} \chi_{\nu}^{\prime}\left(\tilde{U}_{2}-U_{2}\right)_{x} \\
& +\chi_{\nu} \cdot\left(\tilde{U}_{2}\right)_{x x}+\left(1-\chi_{\nu}\right)\left(U_{2}\right)_{x x}
\end{aligned}
$$

Therefore, we find that $\hat{U}_{2}$ satisfies

$$
\left(\hat{U}_{2}\right)_{t}-\nu\left(\hat{U}_{2}\right)_{x x}+\left(F\left(\hat{U}_{2}\right)\right)_{x}=I_{1}+I_{2}+I_{3}
$$

where $I_{k}(k=1,2,3)$ are the collections of like-terms according to whether or not their supports are contained in a same sub-domain of $\mathbb{R}$, more precisely, they are defined by

$$
\begin{aligned}
& I_{1}=\chi_{\nu}\left(\left(\tilde{U}_{2}\right)_{t}-\nu\left(\tilde{U}_{2}\right)_{x x}+f\left(\hat{U}_{2}\right) \cdot\left(\tilde{U}_{2}\right)_{x}\right) \\
& I_{2}=\left(1-\chi_{\nu}\right)\left(\left(U_{2}\right)_{t}-\nu\left(U_{2}\right)_{x x}+f\left(\hat{U}_{2}\right)\left(U_{2}\right)_{x}\right) \\
& I_{3}=\left(\tilde{U}_{2}-U_{2}\right)\left(\frac{d_{t} \chi_{\nu}^{\prime}}{\nu^{\gamma}}-\frac{\chi_{\nu}^{\prime \prime}}{\nu^{2 \gamma-1}}+\frac{\chi_{\nu}^{\prime} f\left(\hat{U}_{2}\right)}{\nu^{\gamma}}\right)-\frac{2 \chi_{\nu}^{\prime}}{\nu^{\gamma-1}}\left(\tilde{U}_{2}-U_{2}\right)_{x} .
\end{aligned}
$$

It is easy to see that the support of $I_{1}, I_{2}$ is, respectively, a subset of $\left[-2 \nu^{\gamma}, 2 \nu^{\gamma}\right]$ and a subset of $\left(-\infty, \nu^{\gamma}\right] \cup\left[\nu^{\gamma}, \infty\right)$, while the support of $I_{3}$ is a subset of $\left[\nu^{\gamma}, 2 \nu^{\gamma}\right] \cup\left[-2 \nu^{\gamma},-\nu^{\gamma}\right]$.

Now we turn to estimate $I_{1}, I_{2}, I_{3}$. Firstly we handle $I_{3}$. In this case one can apply the matching conditions $(3.76)-(3.78)$ and use Taylor expansions to obtain

$$
\partial_{x}^{l}\left(\tilde{U}_{2}-U_{2}\right)(x, t)=O(1) \nu^{(3-l) \gamma}
$$

on the domain $\left\{(x, t)\left|\nu^{\gamma} \leq\right| x-\varphi(t) \mid \leq 2 \nu^{\gamma}, 0 \leq t \leq T\right\}$ and $l=0,1,2,3$. From these estimates (4.12), which can also be found, e.g. in Goodman and Xin [21], the following assertion follows easily that

$$
I_{3}=O(1) \nu^{2 \gamma} \text { as } \nu \rightarrow 0
$$

Moreover, we have

$$
\int_{\mathbb{R}}\left|I_{3}(x, t)\right|^{2} d x=\int_{\left\{\nu^{\gamma} \leq|x-\varphi(t)| \leq 2 \nu^{\gamma}\right\}}\left|I_{3}(x, t)\right|^{2} d x \leq C \nu^{5 \gamma} .
$$

To deal with $I_{1}, I_{2}$ we rearrange the terms of $I_{1}, I_{2}$ as follows

$$
\begin{aligned}
I_{1} & =\chi_{\nu}\left(\left(\tilde{U}_{2}\right)_{t}-\nu\left(\tilde{U}_{2}\right)_{x x}+\left(F\left(\tilde{U}_{2}\right)\right)_{x}\right)+\chi_{\nu} \cdot\left(f\left(\hat{U}_{2}\right)-f\left(\tilde{U}_{2}\right)\right)\left(\tilde{U}_{2}\right)_{x} \\
& =I_{1 a}+I_{1 b} \\
I_{2} & =\left(1-\chi_{\nu}\right)\left(\left(U_{2}\right)_{t}-\nu\left(U_{2}\right)_{x x}+\left(F\left(U_{2}\right)\right)_{x}\right)+\left(1-\chi_{\nu}\right)\left(f\left(\hat{U}_{2}\right)-f\left(U_{2}\right)\right)\left(U_{2}\right)_{x} \\
& =I_{2 a}+I_{2 b} .
\end{aligned}
$$


Moreover, $I_{1 b}$ can be rewritten as

$$
I_{1 b}=\chi_{\nu} \cdot\left(\hat{U}_{2}-\tilde{U}_{2}\right)\left(\tilde{U}_{2}\right)_{x} \cdot \int_{0}^{1} f^{\prime}\left(s \hat{U}_{2}+(1-s) \tilde{U}_{2}\right) d s .
$$

Note that $\operatorname{supp} I_{1 b} \subset\left\{(x, t) \in Q_{T}|| x-\varphi(t) \mid \leq 2 \nu^{\gamma}\right\}$ and $\hat{U}_{2}(x, t)=\tilde{U}_{2}(x, t)$ if $\{(x, t) \in$ $\left.Q_{T}|| x-\varphi(t) \mid \leq \nu^{\gamma}\right\}$. Therefore, from (4.12) and (4.17) we obtain

$$
\left|I_{1 b}\right| \leq \frac{C}{\nu}\left|\left(\hat{U}_{2}-\tilde{U}_{2}\right)\left(\tilde{U}_{2}\right)^{\prime}\right|=O\left(\nu^{3 \gamma-1}\right),
$$

where we used the assumption $\gamma>\frac{2}{3}$ so that $3 \gamma-1>0$. Note, however, that it is enough to assume that $\gamma>\frac{1}{3}$ for the purpose in this sub-section, i.e. the approximate solutions satisfy the corresponding equations. But this assumption will be needed in the stability analysis later in sub-Section 4.2 .

Recalling the construction of $\tilde{U}_{2}$, from equations $(3.73)$ - (3.75), we can rewrite $I_{1 a}$ in the following form

$$
\begin{aligned}
I_{1 a}= & \chi_{\nu} \cdot\left(\left(\tilde{U}_{2}\right)_{t}-\nu\left(\tilde{U}_{2}\right)_{x x}+\left(F\left(\tilde{u}_{0}\right)+\nu f\left(\tilde{u}_{0}\right) \tilde{u}_{1}+\nu^{2}\left(f\left(\tilde{u}_{0}\right) \tilde{u}_{2}+\frac{1}{2} f^{\prime}\left(\tilde{u}_{0}\right) \tilde{u}_{1}^{2}\right)\right)_{x}\right) \\
& +\chi_{\nu} \cdot\left(R_{\tilde{u}}\right)_{x},
\end{aligned}
$$

where the remainder $R_{\tilde{u}}$ is defined by

$$
R_{\tilde{u}}=F\left(\tilde{U}_{2}\right)-\left(F\left(\tilde{u}_{0}\right)+\nu f\left(\tilde{u}_{0}\right) \tilde{u}_{1}+\nu^{2}\left(f\left(\tilde{u}_{0}\right) \tilde{u}_{2}+\frac{1}{2} f^{\prime}\left(\tilde{u}_{0}\right) \tilde{u}_{1}^{2}\right)\right)=O\left(\nu^{3}\right) .
$$

Thus

$$
\left|I_{1 a}\right| \leq\left|\left(R_{\tilde{u}}\right)_{x}\right|=\frac{1}{\nu}\left|\left(R_{\tilde{u}}\right)^{\prime}\right|+O\left(\nu^{2}\right)=O\left(\nu^{2}\right) .
$$

In a similar manner, we now handle $I_{2}$, and rewrite $I_{2 b}$ as

$$
I_{2 b}=\left(1-\chi_{\nu}\right)\left(\hat{U}_{2}-U_{2}\right)\left(U_{2}\right)_{x} \cdot \int_{0}^{1} f^{\prime}\left(s \hat{U}_{2}+(1-s) U_{2}\right) d s .
$$

It is easy to see that $\operatorname{supp} I_{2 b} \subset\left\{|d| \geq \nu^{\gamma}\right\}$ and $\hat{U}_{2}=U_{2}$ if $|d| \geq 2 \nu^{\gamma}$. From the fact that $\hat{U}_{2}-U_{2}=\chi_{\nu}\left(\tilde{U}_{2}-U_{2}\right)$ and (4.12) it follows that

$$
\left|I_{2 b}\right| \leq C \chi_{\nu}\left|\left(\tilde{U}_{2}-U_{2}\right)\left(U_{2}\right)_{x}\right|=O\left(\nu^{3 \gamma}\right)
$$

As for $I_{2 a}$, invoking equations (3.32) and (3.33) we assert that there holds

$$
\begin{aligned}
I_{2 a} & =\left(1-\chi_{\nu}\right)\left(\left(U_{2}\right)_{t}-\nu\left(U_{2}\right)_{x x}+\left(F\left(u_{0}\right)+\nu f\left(u_{0}\right) u_{1}+R_{u}\right)_{x}\right) \\
& =\left(1-\chi_{\nu}\right)\left(R_{u}\right)_{x}+O(\nu) .
\end{aligned}
$$

and the remainder $R_{u}$ is given by

$$
R_{u}=F\left(U_{2}\right)-\left(F\left(u_{0}\right)+\nu f\left(u_{0}\right) u_{1}\right)=O\left(\nu^{2}\right),
$$


hence

$$
I_{2 a}=O(\nu) .
$$

On the other hand, from (4.18) and (4.19) we further obtain

$$
\begin{aligned}
\int_{\mathbb{R}}\left|I_{1}(x, t)\right|^{2} d x & =\int_{\left\{|x-\varphi(t)| \leq 2 \nu^{\gamma}\right\}}\left|I_{1}(x, t)\right|^{2} d x \\
& \leq \int_{\left\{|x-\varphi(t)| \leq \nu^{\gamma}\right\}}\left|I_{1 a}(x, t)\right|^{2} d x+\int_{\left\{\nu^{\gamma} \leq|x-\varphi(t)| \leq 2 \nu^{\gamma}\right\}}\left|I_{1 b}(x, t)\right|^{2} d x \\
& \leq C\left(\nu^{2 * 2+\gamma}+\nu^{6 \gamma-2+\gamma}\right) \leq C \nu^{\gamma} .
\end{aligned}
$$

Here we used the simple inequalities: $6 \gamma-2<4$ and $6 \gamma-2>0$ since we assume $\gamma \in\left(\frac{1}{3}, 1\right)$

From (4.21) and (4.23) it follows that

$$
\begin{aligned}
\int_{\mathbb{R}}\left|I_{2}(x, t)\right|^{2} d x & =\int_{\left\{|x-\varphi(t)| \geq \nu^{\gamma}\right\}}\left|I_{2}(x, t)\right|^{2} d x \\
& \leq C\left(\nu^{2}+\nu^{6 \gamma}\right) \leq C \nu^{\min \{2,6 \gamma\}} \\
& \leq C \nu^{2} .
\end{aligned}
$$

In conclusion, from (4.8), (4.13), (4.18), (4.19), (4.21) and (4.23) we are in a position to assert that $\hat{U}_{2}^{\nu}$ satisfies the equation in the following sense

$$
\left(\hat{U}_{2}^{\nu}\right)_{t}-\nu\left(\hat{U}_{2}^{\nu}\right)_{x x}+\left(F\left(\hat{U}_{2}^{\nu}\right)\right)_{x}=O\left(\nu^{\alpha}\right),
$$

as $\nu \rightarrow 0$. Here $\alpha=3 \gamma-1$ and we used the fact that $3 \gamma-1<2 \gamma<2$ by assumption $\gamma<1$. Furthermore, from construction we see easily that the initial data is satisfied asymptotically too.

Part 2. We now turn to investigate the convergence of $\hat{V}_{2}$. Similar computations show that $\hat{V}_{2}$ can be written in terms of $\tilde{V}_{2}, V_{2}$ as

$$
\begin{aligned}
\left(\hat{V}_{2}\right)_{t}= & \frac{d_{t}}{\nu^{\gamma}} \chi_{\nu}^{\prime}\left(\tilde{V}_{2}-V_{2}\right)+\chi_{\nu} \cdot\left(\tilde{V}_{2}\right)_{t}+\left(1-\chi_{\nu}\right)\left(V_{2}\right)_{t} \\
\left(\hat{V}_{2}\right)_{x}= & \frac{1}{\nu^{\gamma}} \chi_{\nu}^{\prime}\left(\tilde{V}_{2}-V_{2}\right)+\chi_{\nu} \cdot\left(\tilde{V}_{2}\right)_{x}+\left(1-\chi_{\nu}\right)\left(V_{2}\right)_{x} \\
\left(\hat{V}_{2}\right)_{x x}= & \frac{1}{\nu^{2 \gamma}} \chi_{\nu}^{\prime \prime}\left(\tilde{V}_{2}-V_{2}\right)+\frac{2}{\nu^{\gamma}} \chi_{\nu}^{\prime}\left(\tilde{V}_{2}-V_{2}\right)_{x} \\
& +\chi_{\nu} \cdot\left(\tilde{V}_{2}\right)_{x x}+\left(1-\chi_{\nu}\right)\left(V_{2}\right)_{x x},
\end{aligned}
$$

and $\hat{V}_{2}$ satisfies the following equation

$$
\left(\hat{V}_{2}\right)_{t}-\nu\left(\hat{V}_{2}\right)_{x x}+\left(f\left(\hat{U}_{2}\right) \hat{V}_{2}\right)_{x}=J_{1}+J_{2}+J_{3},
$$

where $J_{k}(k=1,2,3)$ are given, according to their supports, by

$$
\begin{aligned}
& J_{1}=\chi_{\nu}\left(\left(\tilde{V}_{2}\right)_{t}-\nu\left(\tilde{V}_{2}\right)_{x x}+\left(f\left(\hat{U}_{2}\right) \tilde{V}_{2}\right)_{x}\right) \\
& J_{2}=\left(1-\chi_{\nu}\right)\left(\left(V_{2}\right)_{t}-\nu\left(V_{2}\right)_{x x}+\left(f\left(\hat{U}_{2}\right) V_{2}\right)_{x}\right) \\
& J_{3}=\left(\frac{d_{t} \chi_{\nu}^{\prime}}{\nu^{\gamma}}-\frac{\chi_{\nu}^{\prime \prime}}{\nu^{2 \gamma-1}}+\frac{f\left(\hat{U}_{2}\right) \chi_{\nu}^{\prime}}{\nu^{\gamma}}\right)\left(\tilde{V}_{2}-V_{2}\right)-\frac{2 \chi_{\nu}^{\prime}}{\nu^{\gamma-1}}\left(\tilde{V}_{2}-V_{2}\right)_{x} .
\end{aligned}
$$


Since for $\hat{V}_{2}$, we also have the same estimate (4.12) which is valid for $\hat{U}_{2}$, namely we have

$$
\partial_{x}^{l}\left(\tilde{V}_{2}-V_{2}\right)=O(1) \nu^{(3-l) \gamma}
$$

on the domain $\left\{(x, t)\left|\nu^{\gamma} \leq\right| x-\varphi(t) \mid \leq 2 \nu^{\gamma}, 0 \leq t \leq T\right\}$ and $l=0,1,2,3$. Thus it follows from (4.33) and the uniform boundedness of $\hat{U}_{2}$ that

$$
J_{3}=O(1) \nu^{2 \gamma}, \text { as } \nu \rightarrow 0
$$

The investigation of convergence for $J_{1}, J_{2}$ is more technically complicated than $I_{1}, I_{2}$. Rewrite $J_{1}, J_{2}$ as

$$
\begin{aligned}
J_{1} & =\chi_{\nu}\left(\left(\tilde{V}_{2}\right)_{t}-\nu\left(\tilde{V}_{2}\right)_{x x}+\left(f\left(\tilde{U}_{2}\right) \tilde{V}_{2}\right)_{x}\right)+\chi_{\nu} \cdot\left(\left(f\left(\hat{U}_{2}\right)-f\left(\tilde{U}_{2}\right)\right) \tilde{V}_{2}\right)_{x} \\
& =J_{1 a}+J_{1 b}
\end{aligned}
$$

and

$$
\begin{aligned}
J_{2} & =\left(1-\chi_{\nu}\right)\left(\left(V_{2}\right)_{t}-\nu\left(V_{2}\right)_{x x}+\left(f\left(U_{2}\right) V_{2}\right)_{x}\right)+\left(1-\chi_{\nu}\right)\left(\left(f\left(\hat{U}_{2}\right)-f\left(U_{2}\right) V_{2}\right)_{x}\right. \\
& =J_{2 a}+J_{2 b} .
\end{aligned}
$$

We now deal with $J_{1 b}$ which can be changed to

$$
\begin{aligned}
J_{1 b}= & \chi_{\nu}\left(\int_{0}^{1} f^{\prime}\left(s \hat{U}_{2}+(1-s) \tilde{U}_{2}\right) d s\left(\hat{U}_{2}-\tilde{U}_{2}\right) \tilde{V}_{2}\right)_{x} \\
= & \left.\chi_{\nu}\left(\int_{0}^{1} f^{\prime}\left(s \hat{U}_{2}\right)+(1-s) \tilde{U}_{2}\right) d s \tilde{V}_{2}\right)_{x}\left(\hat{U}_{2}-\tilde{U}_{2}\right) \\
& \left.+\chi_{\nu} \int_{0}^{1} f^{\prime}\left(s \hat{U}_{2}\right)+(1-s) \tilde{U}_{2}\right) d s\left(\hat{U}_{2}-\tilde{U}_{2}\right)_{x} \tilde{V}_{2} \\
= & O\left(\nu^{3 \gamma-1}\right)+O\left(\nu^{2 \gamma}\right)=O\left(\nu^{3 \gamma-1}\right),
\end{aligned}
$$

here we used the fact that $3 \gamma-1<2 \gamma$ since $\gamma<1$.

Rewriting $J_{1 a}$ as

$$
\begin{aligned}
J_{1 a}= & \chi_{\nu}\left(\left(\tilde{V}_{2}\right)_{t}-\nu\left(\tilde{V}_{2}\right)_{x x}+\left(\left(f\left(\tilde{u}_{0}\right)+f^{\prime}\left(\tilde{u}_{0}\right)\left(\nu \tilde{u}_{1}+\nu^{2} \tilde{u}_{2}\right)+\frac{1}{2} f^{\prime \prime}\left(\tilde{u}_{0}\right)\left(\nu \tilde{u}_{1}\right)^{2}\right) \tilde{V}_{2}\right)_{x}\right) \\
& +\chi_{\nu}\left(R_{\tilde{v}} \tilde{V}_{2}\right)_{x} \\
= & \chi_{\nu}\left(R_{\tilde{v}} \tilde{V}_{2}\right)_{x}+O\left(\nu^{2}\right) .
\end{aligned}
$$

Here, equations (3.90) - (3.92) were used. And the remainder $R_{\tilde{v}}$ is

$$
R_{\tilde{v}}=f\left(\tilde{U}_{2}\right)-\left(f\left(\tilde{u}_{0}\right)+f^{\prime}\left(\tilde{u}_{0}\right)\left(\nu \tilde{u}_{1}+\nu^{2} \tilde{u}_{2}\right)+\frac{1}{2} f^{\prime \prime}\left(\tilde{u}_{0}\right)\left(\nu \tilde{u}_{1}\right)^{2}\right)=O\left(\nu^{3}\right)
$$

Therefore, from (4.38) one has

$$
J_{1 a}=\chi_{\nu}\left(R_{\tilde{v}} \tilde{V}_{2}\right)_{x}=O\left(\nu^{2}\right)
$$


The terms $J_{2 a}, J_{2 b}$ can be estimated in a similar way and we obtain

$$
J_{2 b}=O\left(\nu^{3 \gamma}\right)+O\left(\nu^{2 \gamma}\right)=O\left(\nu^{2 \gamma}\right),
$$

and

$$
\begin{aligned}
J_{2 a} & =\left(1-\chi_{\nu}\right)\left(\left(V_{2}\right)_{t}-\nu\left(V_{2}\right)_{x x}+\left(\left(f\left(u_{0}\right)+\nu f^{\prime}\left(u_{0}\right) u_{1}+R_{v}\right) V_{2}\right)_{x}\right) \\
& \left.=\left(1-\chi_{\nu}\right)\left(O\left(\nu^{2}\right)+R_{v} V_{2}\right)_{x}\right)
\end{aligned}
$$

where $R_{v}$ is given by

$$
R_{v}=f\left(U_{2}\right)-\left(f\left(u_{0}\right)+\nu f^{\prime}\left(u_{0}\right) u_{1}\right)=O\left(\nu^{2}\right) .
$$

It is easy to see that

$$
J_{2 a}=O\left(\nu^{2}\right)
$$

On the other hand, we have the following estimates of integral type

$$
\begin{aligned}
\int_{\mathbb{R}}\left|J_{1}(x, t)\right|^{2} d x & =\int_{\left\{|x-\varphi(t)| \leq 2 \nu^{\gamma}\right\}}\left|J_{1}(x, t)\right|^{2} d x \\
& \leq \int_{\left\{|x-\varphi(t)| \leq \nu^{\gamma}\right\}}\left|J_{1 a}(x, t)\right|^{2} d x+\int_{\left\{\nu^{\gamma} \leq|x-\varphi(t)| \leq 2 \nu^{\gamma}\right\}}\left|J_{1 b}(x, t)\right|^{2} d x \\
& \leq C\left(\nu^{2 * 2+\gamma}+\nu^{6 \gamma-2+\gamma}\right) \leq C \nu^{\gamma}
\end{aligned}
$$

and

$$
\int_{\mathbb{R}}\left|J_{2}(x, t)\right|^{2} d x=\int_{\left\{|x-\varphi(t)| \geq \nu^{\gamma}\right\}}\left|J_{2}(x, t)\right|^{2} d x \leq C\left(\nu^{2 * 2}+\nu^{4 \gamma}\right) \leq C \nu^{\gamma}
$$

Therefore, it follows from (4.29), (4.34), (4.37), (4.39), (4.40) and (4.41) that $\hat{V}_{2}^{\nu}$ satisfies the equation in the following sense

$$
\left(\hat{V}_{2}^{\nu}\right)_{t}-\nu\left(\hat{V}_{2}^{\nu}\right)_{x x}+\left(f\left(\hat{U}_{2}^{\nu}\right) \hat{V}_{2}^{\nu}\right)_{x}=O\left(\nu^{3 \gamma-1}\right),
$$

as $\nu \rightarrow 0$. By construction, the initial data is satisfied asymptotically as well.

Part 3. Finally we turn to investigate the convergence of $\hat{P}_{2}$. Computations show that the derivatives of $\hat{P}_{2}$ can be written in terms of $\tilde{P}_{2}, P_{2}$ as

$$
\begin{aligned}
\left(\hat{P}_{2}\right)_{t}= & \frac{d_{t}}{\nu^{\gamma}} \chi_{\nu}^{\prime}\left(\tilde{P}_{2}-P_{2}\right)+\chi_{\nu} \cdot\left(\tilde{P}_{2}\right)_{t}+\left(1-\chi_{\nu}\right)\left(P_{2}\right)_{t} \\
\left(\hat{P}_{2}\right)_{x}= & \frac{1}{\nu^{\gamma}} \chi_{\nu}^{\prime}\left(\tilde{P}_{2}-P_{2}\right)+\chi_{\nu} \cdot\left(\tilde{P}_{2}\right)_{x}+\left(1-\chi_{\nu}\right)\left(P_{2}\right)_{x} \\
\left(\hat{P}_{2}\right)_{x x}= & \frac{1}{\nu^{2 \gamma}} \chi_{\nu}^{\prime \prime}\left(\tilde{P}_{2}-P_{2}\right)+\frac{2}{\nu^{\gamma}} \chi_{\nu}^{\prime}\left(\tilde{P}_{2}-P_{2}\right)_{x} \\
& +\chi_{\nu} \cdot\left(\tilde{P}_{2}\right)_{x x}+\left(1-\chi_{\nu}\right)\left(P_{2}\right)_{x x}
\end{aligned}
$$

and $\hat{P}_{2}$ satisfies the following equation

$$
-\left(\hat{P}_{2}\right)_{t}-\nu\left(\hat{P}_{2}\right)_{x x}-f\left(\hat{U}_{2}\right)\left(\hat{P}_{2}\right)_{x}=K_{1}+K_{2}+K_{3}
$$


where $K_{i}(i=1,2,3)$ are given, according to their supports, by

$$
\begin{aligned}
& K_{1}=-\chi_{\nu}\left(\left(\tilde{P}_{2}\right)_{t}+\nu\left(\tilde{P}_{2}\right)_{x x}+f\left(\hat{U}_{2}\right)\left(\tilde{P}_{2}\right)_{x}\right), \\
& K_{2}=-\left(1-\chi_{\nu}\right)\left(\left(P_{2}\right)_{t}+\nu\left(P_{2}\right)_{x x}+f\left(\hat{U}_{2}\right)\left(P_{2}\right)_{x}\right) \\
& K_{3}=-\left(\frac{d_{t} \chi_{\nu}^{\prime}}{\nu^{\gamma}}+\frac{\chi_{\nu}^{\prime \prime}}{\nu^{2 \gamma-1}}+\frac{f\left(\hat{U}_{2}\right) \chi_{\nu}^{\prime}}{\nu^{\gamma}}\right)\left(\tilde{P}_{2}-P_{2}\right)-\frac{2 \chi_{\nu}^{\prime}}{\nu^{\gamma-1}}\left(\tilde{P}_{2}-P_{2}\right)_{x} .
\end{aligned}
$$

By similar arguments as done for $\hat{U}_{2}^{\nu}$, we prove that

$$
-\left(\hat{P}_{2}^{\nu}\right)_{t}-\nu\left(\hat{P}_{2}^{\nu}\right)_{x x}-f\left(\hat{U}_{2}^{\nu}\right)\left(\hat{P}_{2}^{\nu}\right)_{x}=O\left(\nu^{3 \gamma-1}\right),
$$

as $\nu \rightarrow 0$. We omit the details here.

Thus from parts 1, 2 and 3 we see that the proof of Theorem 3.1 is complete.

\subsection{Proof of the convergence}

This sub-section is devoted to the proof of Theorem 4.1. Since this sub-section is concerned with the proof of convergence as $\nu \rightarrow 0$, we denote $\hat{U}_{2}^{\nu}, \hat{V}_{2}^{\nu}, \hat{P}_{2}^{\nu}$ by $\hat{U}^{\nu}, \hat{V}^{\nu}, \hat{P}^{\nu}$, respectively, for the sake of simplicity. We begin with the following lemma.

Lemma 4.2 For $\eta$ defined in (4.4),

$$
\sup _{0 \leq t \leq T}\left(\left\|u^{\nu}(\cdot, t)-u(\cdot, t)\right\|+\left\|v^{\nu}(\cdot, t)-v(\cdot, t)\right\|+\left\|p^{\nu}(\cdot, t)-p(\cdot, t)\right\|\right) \leq C \nu^{\eta},
$$

as $\nu \rightarrow 0$. Here $u^{\nu}, u, v^{\nu}, v, p^{\nu}, p$ are the same as in Theorem 4.1.

Proof. The proof of Lemma 4.2 consists of two steps.

Step 1. By construction of the approximate solutions, we conclude that

i) for $(x, t) \in\left\{|x-\varphi(t)| \geq 2 \nu^{\gamma}, 0 \leq t \leq T\right\}$,

$$
\hat{U}^{\nu}(x, t)=u(x, t)+O(1) \nu
$$

where $O(1)$ denotes a function which is square-integrable over outer region by the argument in sub-Section 4.1.

ii) for $(x, t) \in\left\{|x-\varphi(t)| \leq \nu^{\gamma}\right\}$,

$$
\hat{U}^{\nu}(x, t)=\tilde{u}_{0}\left(r_{\nu}, t\right)+O(1) \nu,
$$

iii) and for $(x, t) \in\left\{\nu^{\gamma} \leq|x-\varphi(t)| \leq 2 \nu^{\gamma}\right\}$,

$$
\hat{U}^{\nu}(x, t)=U^{\nu}(x, t)+\chi_{\nu}(x, t)\left(\tilde{U}^{\nu}(x, t)-U^{\nu}(x, t)\right) \sim U^{\nu}(x, t)+O(1) \nu^{3 \gamma} .
$$

Here we have used again the estimate $\tilde{U}_{2}^{\nu}(x, t)-U_{2}^{\nu}(x, t)=O(1) \nu^{3 \gamma}$. We can also establish similar estimates for $\hat{V}^{\nu}, \hat{P}^{\nu}$. Therefore, one can obtain

$$
\begin{aligned}
\sup _{0 \leq t \leq T}\left\|u(\cdot, t)-\hat{U}^{\nu}(\cdot, t)\right\|^{2}+ & \sup _{0 \leq t \leq T}\left\|v(\cdot, t)-\hat{V}^{\nu}(\cdot, t)\right\|^{2} \leq C \nu^{\min \{3 \gamma, 2\}}, \\
\sup _{0 \leq t \leq T}\left\|p_{n}(\cdot, t)-\hat{P}^{\nu}(\cdot, t)\right\|^{2} & \leq C \nu^{\min \{3 \gamma, 2\}}
\end{aligned}
$$


where $p_{n}$ is the reversible solution to the inviscid adjoint equation $-\partial_{t} p-f(u) \partial_{x} p=0$ with final data $p(x, T)=p^{T}(x)$.

Step 2. Next we need to estimate $\sup _{0 \leq t \leq T}\left\|u^{\nu}(\cdot, t)-\hat{U}^{\nu}(\cdot, t)\right\|^{2}, \sup _{0 \leq t \leq T} \| v^{\nu}(\cdot, t)-$ $\hat{V}^{\nu}(\cdot, t) \|^{2}$ and $\sup _{0 \leq t \leq T}\left\|p^{\nu}(\cdot, t)-\hat{P}^{\nu}(\cdot, t)\right\|^{2}$. To this end, we will employ the $L^{2}$-energy method. Suppose that we have obtained

$$
\sup _{0 \leq t \leq T}\left\|u^{\nu}(\cdot, t)-\hat{U}^{\nu}(\cdot, t)\right\|^{2} \leq C \nu^{\eta_{1}}
$$

where $\eta_{1}=\gamma+1$. Then we arrive at (4.51) by using the triangle inequality, the estimate (4.52) and the fact that

$$
\min \left\{\frac{3}{2} \gamma, \frac{1+\gamma}{2}, 1\right\}=\frac{1+\gamma}{2}
$$

since $\frac{2}{3}<\gamma<1$. So one concludes for $v, p$. This step consists of three parts.

Part 1. Firstly we prove (4.54). Define

$$
\bar{w}(x, t)=u^{\nu}(x, t)-\hat{U}^{\nu}(x, t),
$$

Then $\bar{w}(x, t)$ satisfies

$$
\begin{aligned}
\bar{w}_{t}-\nu \bar{w}_{x x}+f\left(u^{\nu}\right) \bar{w}_{x} & =\mathcal{F}, \\
\bar{w}(x, 0) & =\bar{w}_{0}(x),
\end{aligned}
$$

where

$$
\begin{aligned}
\mathcal{F} & :=-\sum_{i=1}^{3} I_{i}-Q(x, t)-f^{\prime}\left(\hat{U}^{\nu}\right) \bar{w} \hat{U}_{x}^{\nu} \\
Q & :=\left(f\left(u^{\nu}\right)-f\left(\hat{U}^{\nu}\right)-f^{\prime}\left(\hat{U}^{\nu}\right) \bar{w}\right) \hat{U}_{x}^{\nu}
\end{aligned}
$$

Rescaling as follows

$$
\bar{w}(x, t)=\nu w(y, \tau), \text { where } y=\frac{x-\varphi(t)}{\nu}, \text { and } \tau=\frac{t}{\nu},
$$

one has

$$
\bar{w}_{t}(x, t)=w_{\tau}(y, \tau)-\varphi^{\prime}(\nu \tau) w_{y}, \quad \bar{w}_{x}(x, t)=w_{y}(y, \tau), \quad \bar{w}_{x x}(x, t)=\frac{1}{\nu} w_{y y}(y, \tau),
$$

and problem $(4.55)$ - (4.56) turns out to be

$$
\begin{aligned}
w_{\tau}-w_{y y}+\left(f\left(u^{\nu}\right)-\varphi^{\prime}(\nu \tau)\right) w_{y} & =\mathcal{F}(\nu y+\varphi, \nu \tau), \\
w(x, 0) & =w_{0}(x) .
\end{aligned}
$$

Here the initial data $w_{0}$ can be chosen very small such that

$$
\left\|w_{0}\right\|_{H^{1}(\mathbb{R})}^{2} \leq C \nu \leq C \nu^{\gamma}
$$

provided that $\nu$ is suitably small.

The existence of solution $w \in C\left([0, T / \nu] ; H^{1}(\mathbb{R})\right)$ to problem (4.59) - (4.60) follows from the method of continuation of local solution, which is based on local existence of solution and a priori estimates stated in the following proposition 
Proposition 4.3 (A priori estimates) Suppose that problem (4.59) - (4.60) has a solution $w \in C\left(\left[0, \tau_{0}\right] ; H^{1}(\mathbb{R})\right)$ for some $\tau_{0} \in(0, T / \nu]$. There exist positive constants $\mu_{1}, \nu_{1}$ and $C$, which are independent of $\nu, \tau_{0}$, such that if

$$
\nu \in\left(0, \nu_{1}\right], \sup _{0 \leq \tau \leq \tau_{0}}\|w(\tau, \cdot)\|_{H^{1}(\mathbb{R})}+\mu_{0} \leq \mu_{1}
$$

then

$$
\sup _{0 \leq \tau \leq \tau_{0}}\|w(\tau, \cdot)\|_{H^{1}(\mathbb{R})}^{2}+\int_{0}^{\tau_{0}}\|w(\tau, \cdot)\|_{H^{2}(\mathbb{R})}^{2} d \tau \leq C \nu^{\gamma} .
$$

Proof. The proof of this proposition is divided into three steps.

Step 1. By the maximum principle and construction of approximate solution $\hat{U}^{\nu}$, we have

$$
\left\|u^{\nu}\right\|_{L^{\infty}\left(Q_{\tau_{0}}\right)} \leq C, \quad\left\|\hat{U}^{\nu}\right\|_{L^{\infty}\left(Q_{\tau_{0}}\right)} \leq C .
$$

Whence, from the smallness assumption (4.61) and definition of $Q$ it follows that

$$
|Q(x, t)| \leq C\left|\bar{w}^{2} \hat{U}_{x}^{\nu}\right|
$$

Step 2. Multiplying eq. (4.59) by $w$ and integrating the resulting equation with respect to $y$ over $\mathbb{R}$ we obtain

$$
\frac{1}{2} \frac{d}{d \tau}\|w\|^{2}+\left\|w_{y}\right\|^{2}+\int_{\mathbb{R}}\left(f\left(u^{\nu}\right)-\varphi^{\prime}(\nu \tau)\right) w_{y} w d y=\int_{\mathbb{R}} \mathcal{F}(\nu y+\varphi, \nu \tau) w d y
$$

We first deal with the term

$$
\int_{\mathbb{R}} f^{\prime}\left(\hat{U}^{\nu}\right) \bar{w} \hat{U}_{x}^{\nu} w d y=\int_{\mathbb{R}} f^{\prime}\left(\hat{U}^{\nu}\right) w^{2} \nu \hat{U}_{x}^{\nu} d y
$$

From the property of profile $\hat{U}$ we have

$$
\nu \hat{U}_{x}^{\nu}=\hat{U}_{y}^{\nu} \rightarrow 0, \text { as } \nu \rightarrow 0
$$

Thus

$$
\left|\int_{\mathbb{R}} f^{\prime}\left(\hat{U}^{\nu}\right) \bar{w} \hat{U}_{x}^{\nu} w d y\right| \leq C\|w\|^{2} .
$$

For the term of $Q$, it is easier to obtain that

$$
\left|\int_{\mathbb{R}} Q(y, \tau) \hat{U}_{x}^{\nu} w d y\right| \leq C \int_{\mathbb{R}}\left|\bar{w}^{2} \hat{U}_{x}^{\nu} w\right| d y \leq C\|w\|^{2} .
$$

It remains to deal with the term $\int_{\mathbb{R}} I w d y$ where $I=\sum_{i=1}^{3} I_{i}$. We invoke the $L^{2}$-norm estimates for $I$, i.e. (4.24) and (4.25), which have been obtained in sub-Section 3.1, and get

$$
\begin{aligned}
\left|\int_{0}^{\tau} \int_{\mathbb{R}} I w d \tau d y\right| & \leq C\left(\int_{0}^{\tau} \int_{\mathbb{R}}|I|^{2} d \tau d y+\int_{0}^{\tau} \int_{\mathbb{R}}|w|^{2} d \tau d y\right) \\
& \leq C \nu^{\gamma}+C \int_{0}^{\tau} \int_{\mathbb{R}}|w|^{2} d \tau d y .
\end{aligned}
$$


Here, we used the assumption that $\gamma>\frac{2}{3}$ to estimate the term $I$.

Finally, by the Young inequality one gets

$$
\left|\int_{\mathbb{R}}\left(f\left(u^{\nu}\right)-\varphi^{\prime}(\nu \tau)\right) w_{y} w d y\right| \leq \frac{1}{2}\left\|w_{y}\right\|^{2}+C\|w\|^{2} .
$$

Therefore, the above arguments and integrating (4.64) with respect to $\tau$ yield

$$
\frac{1}{2}\|w(\tau)\|^{2}+\frac{1}{2} \int_{0}^{\tau}\left\|w_{y}(s)\right\|^{2} d s \leq C \int_{0}^{\tau}\|w(s)\|^{2} d s+C \nu^{\gamma},
$$

from which and the Gronwall inequality in the integral form we then arrive at

$$
\|w(\tau)\|^{2} \leq C \nu^{\gamma}
$$

So we also obtain

$$
\left\|u^{\nu}(\cdot, t)-\hat{U}^{\nu}(\cdot, t)\right\|^{2}=\nu\|w(\tau)\|^{2} \leq C \nu^{1+\gamma} .
$$

Step 3. Next we multiply eq. (4.60) by $-w_{y y}$ and integrate the resultant with respect to $y$ to get

$$
\begin{aligned}
\frac{1}{2} \frac{d}{d \tau}\left\|w_{y}\right\|^{2} & +\left\|w_{y y}\right\|^{2}-\int_{\mathbb{R}}\left(f\left(u^{\nu}\right)-\varphi^{\prime}(\nu \tau)\right) w_{y y} w_{y} d y \\
& =-\int_{\mathbb{R}} \mathcal{F}(\nu y+\varphi, \nu \tau) w_{y y} d y
\end{aligned}
$$

Using (4.62) and estimates on $I_{i}(I=1,2,3)$, from the Young inequality we then arrive at

$$
\frac{1}{2}\left\|w_{y}(\tau)\right\|^{2}+\frac{1}{2} \int_{0}^{\tau}\left\|w_{y y}(s)\right\|^{2} d s \leq C \int_{0}^{\tau}\left\|w_{y}(s)\right\|^{2} d s+C \nu^{\gamma},
$$

making use of Gronwall's inequality again one has

$$
\left\|w_{y}(\tau)\right\|^{2} \leq C \nu^{\gamma}
$$

Part 2. In this part we are in a position to prove the convergence of $p^{\nu}-\hat{P}^{\nu}$, namely to prove

$$
\sup _{0 \leq t \leq T}\left\|p^{\nu}(\cdot, t)-\hat{P}^{\nu}(\cdot, t)\right\|^{2} \leq C \nu^{\eta} .
$$

Let

$$
\bar{q}=p^{\nu}-\hat{P}^{\nu},
$$

then computations yield that $\bar{q}$ satisfies

$$
\begin{aligned}
-\bar{q}_{t}-\nu \bar{q}_{x x}-f\left(u^{\nu}\right) \bar{q}_{x} & =\mathcal{G}, \\
\bar{q}(x, T) & =\bar{q}_{0}(x) .
\end{aligned}
$$


Here

$$
\begin{aligned}
\mathcal{G} & :=-\sum_{i=1}^{3} K_{i}+Q_{1}(x, t)+f^{\prime}\left(\hat{U}^{\nu}\right) \bar{w} \hat{P}_{x}^{\nu} \\
Q_{1} & :=\left(f\left(u^{\nu}\right)-f\left(\hat{U}^{\nu}\right)-f^{\prime}\left(\hat{U}^{\nu}\right) \bar{w}\right) \hat{P}_{x}^{\nu}
\end{aligned}
$$

and $K_{i}$ (with $i=1,2,3$ ) can be defined in a slightly different way as in the arguments for $\hat{U}^{\nu}$.

Rescaling again as follows

$$
\bar{q}(x, t)=\nu q(y, \tau), \text { where } y=\frac{x-\varphi(t)}{\nu}, \text { and } \tau=\frac{T-t}{\nu},
$$

one has

$$
\bar{q}_{t}(x, t)=-q_{\tau}(y, \tau)-\varphi^{\prime}(\nu \tau) q_{y}, \quad \bar{q}_{x}(x, t)=q_{y}(y, \tau), \quad \bar{q}_{x x}(x, t)=\frac{1}{\nu} q_{y y}(y, \tau),
$$

and problem (4.71) - (4.72) can be rewritten as

$$
\begin{aligned}
q_{\tau}-q_{y y}-\left(f\left(u^{\nu}\right)-\varphi^{\prime}(\nu \tau)\right) q_{y} & =\mathcal{G}(\nu y+\varphi, \nu \tau), \\
q(y, 0) & =\bar{q}_{0}(\nu y+\psi(T)) .
\end{aligned}
$$

Employing again the method of continuation of a local solution based upon the a priori estimates, we prove easily the existence of solution $q \in C\left(\left[0, \tau_{0}\right] ; H^{2}(\mathbb{R})\right)$, also we have

$$
\left\|p^{\nu}(\cdot, t)-\hat{P}^{\nu}(\cdot, t)\right\|^{2}=\nu\|q(\tau)\|^{2} \leq C \nu^{1+\gamma} .
$$

Furthermore, we assume that the initial $\left.p\right|_{t=0}$ is bounded in $H^{2}(\mathbb{R})$, similar to the argument in Goodman and Xin [21], we can prove that for any constant $h>0$

$$
\sup _{0 \leq t \leq T}\left\|p^{\nu}-p\right\|_{L^{\infty}\left(\Omega_{h}\right)} \rightarrow 0
$$

as $\nu \rightarrow 0$, here $\Omega_{h}$ is defined by

$$
\left.\Omega_{h}=\left\{(x, t) \in Q_{T}\right\}|| x-\varphi(t) \mid>h\right\} .
$$

Part 3. To prove the convergence of $\bar{O}:=v^{\nu}-\hat{V}_{2}^{\nu}$, we rewrite equations (3.3) and (3.123) as follows

$$
\begin{aligned}
v_{t}^{\nu}-\nu v_{x x}^{\nu}+f\left(u^{\nu}\right)\left(v^{\nu}\right)_{x}+\left(f\left(u^{\nu}\right)\right)_{x} v^{\nu} & =0 \\
\left(\hat{V}_{2}^{\nu}\right)_{t}-\nu\left(\hat{V}_{2}^{\nu}\right)_{x x}+f\left(\hat{U}_{2}^{\nu}\right)\left(\hat{V}_{2}^{\nu}\right)_{x}+\left(f\left(\hat{U}_{2}^{\nu}\right)\right)_{x} \hat{V}_{2}^{\nu} & =\sum_{i=1}^{3} J_{i} .
\end{aligned}
$$

Then we find $\bar{O}$ satisfies

$$
\bar{O}_{t}-\nu \bar{O}_{x x}+f\left(u^{\nu}\right) \bar{O}_{x}+\left(f\left(u^{\nu}\right)\right)_{x} \bar{O}=\mathcal{H}
$$


and

$$
\mathcal{H}=-\left(f\left(u^{\nu}\right)-f\left(\hat{U}_{2}^{\nu}\right)\right)_{x} \hat{V}_{2}^{\nu}-\left(f\left(u^{\nu}\right)-f\left(\hat{U}_{2}^{\nu}\right)\right)\left(\hat{V}_{2}^{\nu}\right)_{x}-\sum_{i=1}^{3} J_{i} .
$$

We use again the rescaling technique as follows

$$
\bar{O}(x, t)=\nu O(y, \tau), \text { where } y=\frac{x-\varphi(t)}{\nu}, \text { and } \tau=\frac{t}{\nu},
$$

To overcome the difficulty, due to the last term in the left hand side of (4.80), in the proof of the convergence of $\bar{O}$, we make use of the convergence result in Part 1 , the estimate $\nu \int_{0}^{t}\left\|u_{x}^{\nu}(\tau)\right\|^{2} d \tau \leq C$, the interpolation inequality in the following form

$$
\|f\|_{L^{4}(\mathbb{R})} \leq C\left\|f_{x}\right\|^{\frac{1}{4}}\|f\|^{\frac{3}{4}},
$$

and the Young inequality of the form: $a b c \leq \varepsilon a^{4}+C_{\varepsilon}\left(b^{4}+c^{2}\right)$. We then estimate

$$
\begin{aligned}
\left|\int_{\mathbb{R}}\left(f\left(u^{\nu}\right)\right)_{x} \bar{O} O d x\right| & \leq C \nu \int_{\mathbb{R}}\left|u_{x}^{\nu}\right| O^{2} d x \\
& \leq C \nu\left\|u_{x}^{\nu}\right\|\|O\|_{L^{4}}^{2} \\
& \leq C \nu\left\|u_{x}^{\nu}\right\|\left(\left\|O_{x}\right\|^{\frac{1}{2}}\|O\|^{\frac{3}{2}}\right) \\
& \leq C\left(\nu^{2}\left\|u_{x}^{\nu}\right\|^{2}\|O\|^{2}+\|O\|^{2}\right)+\frac{1}{2}\left\|O_{x}\right\|^{2} .
\end{aligned}
$$

The last term in the right hand side of (4.81) can be absorbed by the left hand side. The other terms in (4.80) and $\mathcal{H}$ can be treated in a similar way as in Part 1 , and we omit the details.

Therefore, the proof of Theorem 4.1 is complete.

\section{The method of alternating descent directions: Viscous case}

This section is concerned with the extension, for the purpose of numerical computation, of the arguments in sub-Section 2.3 on the choices of alternating descent directions, to the viscous problem. We consider the case that the initial data $u^{I}$ satisfies

$$
\left.\begin{array}{l}
u^{I} \text { is continuous and smooth up to the shock, and } u_{x}^{I} \text { has only a } \\
\text { discontinuity at } \varphi^{I} \text {. Moreover, } u^{I} \text { and } u_{x}^{I} \text { are integrable over } Q_{T} \backslash \Sigma \text {. }
\end{array}\right\}
$$

As pointed out in the end of Section 1, for any positive $\nu$ solutions $\delta u, p$ of equations (1.6) and (1.10) are smooth, thus the Gateaux derivative of the functional $J$ is as follows

$$
\delta J=<\delta J\left(u^{I}\right), \delta u^{I}>=\int_{\mathbb{R}} p(x, 0) \delta u^{I}(x) d x,
$$

where the adjoint state $p=p_{\nu}$ is the solution to (1.10) with initial datum $p(x, T)=$ $u(x, T)-u^{D}(x)$.

To exploit the possibilities that the alternate descent method provides, we take into account, as in [12], the effects of possible infinitesimal perturbations of initial datum and also infinitesimal translations, and choose the initial data $u_{\varepsilon}^{I}$ of the form

$$
u_{\varepsilon}^{I}(x)=u_{\varepsilon}^{I}\left(x+\varepsilon \delta \varphi^{I}\right)+\varepsilon \delta u_{\varepsilon}^{I}(x) .
$$


By a Taylor expansion, (5.3) can be rewritten in the following form

$$
u_{\varepsilon}^{I}(x)=u^{I}(x)+\varepsilon\left(\delta \varphi^{I} u_{x}^{I}(x)+\delta u^{I}(x)\right)+O\left(\varepsilon^{2}\right) .
$$

Correspondingly we formulate the linearized problem as follows

$$
\begin{aligned}
(\delta u)_{t}+(f(u) \delta u)_{x} & =\nu(\delta u)_{x x} \\
\delta u(x, 0) & =\delta \varphi^{I} u_{x}^{I}(x)+\delta u^{I}(x),
\end{aligned}
$$

and its adjoint problem is

$$
\begin{aligned}
-p_{t}-f(u) p_{x} & =\nu p_{x x}, \\
p(x, 0) & =p_{\varepsilon}^{T}(x) .
\end{aligned}
$$

But by doing this way it leads to some difficulties: A Dirac delta appears in the Taylor expansion (5.4) and in the initial data (5.6) provided that $u^{I}(x)$ has a jump. How to understand this expansion and how to solve problem (5.5) - (5.6)? For understanding an expansion with Dirac masses, we refer the reader to e.g. [14]. As for this type of initial value problems, it is difficult even though (5.5) is parabolic for fixed $\nu$. There are not too many references related to this topic: some authors investigate generalized solutions to Burgers' equation with singular data see, e.g. [4], while in [10, 5, 13] the authors studied parabolic equations with a Dirac delta as an initial datum. However the solution exists only for some special cases. Another difficulty is that we need more regular initial data for our construction of asymptotic expansions, moreover, we also consider the limit as $\nu \rightarrow 0$, the limit equation of (5.5) has discontinuous coefficient, which leads to a term like $(f(u) \delta u)_{x}$ where $f(u)$ and $\delta u$ may be discontinuous.

Therefore, we don't expand $u^{I}\left(x+\varepsilon \delta \varphi^{I}\right)$ directly as done in (5.4). To overcome the above difficulties we approximate the initial datum as follows: We use again the cut-off function $\chi_{h}$ for $h>0$, define $\xi=\frac{x-\varphi^{I}}{h}$ and choose $U^{I}$ a smooth function in $\xi$ satisfying the matching conditions

$$
\lim _{\xi \rightarrow \pm \infty} U^{I}(\xi)=\lim _{\xi \rightarrow \varphi^{I} \pm 0} u^{I}(x),
$$

then by a Taylor expansion, we obtain

$$
\begin{aligned}
u_{\varepsilon, h}^{I}(x)= & \chi_{h}(x) U^{I}(\xi)+\left(1-\chi_{h}(x)\right) u^{I}\left(x+\varepsilon \delta \varphi^{I}\right)+\varepsilon \delta u_{\varepsilon}^{I}(x) \\
= & \chi_{h}(x) U^{I}(\xi)+\left(1-\chi_{h}(x)\right) u^{I}(x) \\
& +\varepsilon\left(\left(1-\chi_{h}(x)\right) u_{x}^{I}(x) \delta \varphi^{I}+\delta u_{\varepsilon}^{I}(x)\right)+O\left(\varepsilon^{2}\right) .
\end{aligned}
$$

Letting $h \rightarrow 0$ we see that there is no any Dirac delta appearing in (5.9) any more. The corresponding linearized problem turn out to

$$
\begin{aligned}
(\delta u)_{t}+(f(u) \delta u)_{x} & =\nu(\delta u)_{x x} \\
\delta u(x, 0) & =\delta \varphi^{I}\left(1-\chi_{h}(x)\right) u_{x}^{I}(x)+\delta u^{I}(x)
\end{aligned}
$$

Moreover for any fixed $\nu$ we can easily pass the solution $\delta u_{\nu, h}$ of problem $(5.10)-(5.11)$ to its limit, say $\delta u_{\nu}$, as $h \rightarrow 0$.

We assume, to begin with, that $u^{I}$ satisfies (5.1). We shall make use of the convergence result (4.77), from which one concludes that the smooth solution $p^{\nu}$ of problem (5.7)-(5.8) 
is very close to its limit $p$, provided that $\nu$ is very small. To determine the alternating descent directions, the first thing to be done is to identify the region of influence $\left[x^{-}, x^{+}\right]$ of the inner boundary of the inviscid adjoint system. Similar to [12], we can compute $x^{-}, x^{+}$, so the region of influence is thus defined. Then we need to identify the variations $\left(\delta u^{I}, \delta \varphi^{I}\right)$ such that

$$
\int_{x^{-}}^{x^{+}} p^{\nu}(x, 0)\left(\delta \varphi^{I}\left(1-\chi_{h}(x)\right) u_{x}^{I}(x)+\delta u^{I}(x)\right) d x=0 .
$$

It is easy to see that we can rewrite (5.12) as

$$
\int_{\left[x^{-}, x^{+}\right] \backslash\left\{\varphi^{I}\right\}} p^{\nu}(x, 0)\left(\delta \varphi^{I} u_{x}^{I}(x)+\delta u^{I}(x)\right) d x=o_{h}(1),
$$

where, $o_{h}(1)$ denotes a small quantity such that $o_{h}(1) \rightarrow 0$ as $h \rightarrow 0$.

In [12], it is argued that if $p^{\nu}(x, 0)$ were constant within the interval $\left[x^{-}, x^{+}\right]$as in the inviscid case, this would amount to consider variations such that

$$
\delta \varphi^{I}=\frac{\int_{x^{-}}^{x^{+}} \delta u^{I}(x) d x}{u^{I}\left(x^{+}\right)-u^{I}\left(x^{-}\right)} .
$$

One possibility would be to consider variations $\delta u^{I}$ in $\left[x^{-}, x^{+}\right]$such that $\int_{x^{-}}^{x^{+}} \delta u^{I}(x) d x=0$ and $\delta \varphi^{I}=0$. The variation of the functional $J$ would then be

$$
\delta J=\int_{\left\{x>x^{+}\right\} \cup\left\{x<x^{-}\right\}} p^{\nu}(x, 0) \delta u^{I}(x) d x,
$$

and the optimal descent direction

$$
\delta u^{I}(x)=-p^{\nu}(x, 0) \text {, in }\left\{x>x^{+}\right\} \cup\left\{x<x^{-}\right\} .
$$

However, the assumption that $p^{\nu}(x, 0)$ is constant within the interval $\left[x^{-}, x^{+}\right]$, is not true, in general, in the viscous case, and is only true in the inviscid case. Invoking (4.77), we find $p^{\nu}(x, 0)$ in $(5.13)$ is close to a constant over $\Omega_{\mu}$, provided that $\nu$ is small and $n$ is large. Thus we rewrite (5.13) as follows

$$
\begin{aligned}
o_{h}(1) & =\int_{x^{-}}^{x^{+}} p^{\nu}(x, 0)\left(\delta \varphi^{I} u_{x}^{I}(x)+\delta u^{I}(x)\right) d x \\
& =\int_{\varphi^{I}-\mu}^{\varphi^{I}+\mu}+\int_{\left[x^{-}, x^{+}\right] \backslash\left[\varphi^{I}-\mu, \varphi^{I}+\mu\right]} p^{\nu}(x, 0)\left(\delta \varphi^{I} u_{x}^{I}(x)+\delta u^{I}(x)\right) d x \\
& =I_{1}+I_{2} .
\end{aligned}
$$

Here, $\mu$ is a small positive number. Recalling $\delta u^{I} \in L^{1}(\mathbb{R}) \cap L^{\infty}(\mathbb{R})$, by assumptions (5.1) and the results $p^{\nu} \in L^{\infty}\left(Q_{T}\right)$, we see that the integrand is integrable over $\mathbb{R} \backslash\left\{\varphi^{I}\right\}$, then $I_{1}$ is small and depends on the small parameter $\mu$ (also $n, \nu$, but they are assumed temporarily to be fixed). While for $I_{2}$ we can replace $p^{\nu}$ by $p$, however a small error, depending on $\nu$, appears. Then (5.15) can be rewritten as

$$
\begin{aligned}
0= & C_{h, \nu, \mu}+\int_{\left[x^{-}, x^{+}\right] \backslash\left[\varphi^{I}-\mu, \varphi^{I}+\mu\right]} p(x, 0)\left(\delta \varphi^{I} u_{x}^{I}(x)+\delta u^{I}(x)\right) d x \\
= & C_{h, \nu, \mu}+p(x, 0)\left(u^{I}\left(x^{+}\right)-u^{I}\left(x^{-}\right)-\left(u^{I}\left(\varphi^{I}+\mu\right)-u^{I}\left(\varphi^{I}-\mu\right)\right)\right) \delta \varphi^{I} \\
& +p(x, 0)\left(\int_{\left[x^{-}, x^{+}\right] \backslash\left[\varphi^{I}-\mu, \varphi^{I}+\mu\right]} \delta u^{I}(x) d x\right) .
\end{aligned}
$$


Here $C_{h, \nu, \mu}$ denotes a small quantity depending on $h, \nu, \mu$. But by assumption (5.1), $u^{I}\left(\varphi^{I}+\mu\right)-u^{I}\left(\varphi^{I}-\mu\right) \rightarrow\left[u^{I}\right]_{\varphi^{I}}$ as $\mu \rightarrow 0$. Therefore,

$$
\begin{aligned}
\delta \varphi^{I} & =-\frac{C_{h, \nu, \mu} / p(x, 0)+\int_{\left[x^{-}, x^{+}\right] \backslash\left[\varphi^{I}-\mu, \varphi^{I}+\mu\right]} \delta u^{I}(x) d x}{u^{I}\left(x^{+}\right)-u^{I}\left(x^{-}\right)-\left[u^{I}\right]_{\varphi^{I}}} \\
& \sim-\frac{\int_{\left[x^{-}, x^{+}\right]} \delta u^{I}(x) d x}{u^{I}\left(x^{+}\right)-u^{I}\left(x^{-}\right)-\left[u^{I}\right]_{\varphi^{I}}} .
\end{aligned}
$$

This implies that we can choose a descent direction as the case that $p^{\nu}(x, 0)$ is a constant, at least for numerical simulation since there always are some errors when we compute any quantity. Moreover, we can extend $\delta u^{I}$ to the subdomain $\left[x^{-}, x^{+}\right]$such that

$$
\int_{x^{-}}^{x^{+}} \delta u^{I}(x) d x=0
$$

whence

$$
\delta \varphi^{I}=0
$$

The second class of variations is the one that takes advantage of the infinitesimal translations $\delta \varphi^{I}$. We can then set $\delta u^{I} \equiv 0$ and choose $\delta \varphi^{I}$ such that

$$
\delta \varphi^{I}=-\int_{\mathbb{R} \backslash\left\{\varphi^{I}\right\}} p(x, 0) u_{x}^{I}(x) d x-\left[u^{I}\right]_{\varphi^{I}} p\left(\varphi^{I}, 0\right) .
$$

As mentioned above, we could consider slightly different variations of the initial data of the form

$$
\delta \varphi^{I}=-\left[u^{I}\right]_{\varphi^{I}} p\left(\varphi^{I}, 0\right)
$$

as in [11].

In this way, we have identified two classes of variations and its approximate values inspired in the structure of the state and the adjoint state in the inviscid case, allowing to implement the method of alternating descent in the inviscid case when $u^{I}$ is discontinuous.

The efficiency of the method discussed here has been illustrated by several numerical experiments in the case of the Burgers equation in reference [12], where an implicit assumption that $\sigma$ is large is assumed due to the use of equation (2.6) (corresponding to (3.66) in the case that $\sigma=\infty$ ) with $f(u)=u$. However, with the help of the modified equation (3.66), we can carry out simulations for optimal control problems of nonlinear conservation laws, in the case that $\sigma$ is not too large.

From the above arguments we can draw the following conclusion.

Conclusion 5.1: For numerical simulation, alternating descent method is efficient for suitably small $\nu$; the classical descent method is applicable for suitably large $\nu$. Otherwise both may not work well. More precisely,

i) There exists a number $\nu_{0}$ such that for any $\nu \in\left(0, \nu_{0}\right]$, solution $p(x, 0)$ can be used to replace the exact solution $p^{\nu}(x, 0)$ (as in (5.16)) with a small error which is much smaller than the mesh size. Thus this error can be omitted, and the algorithm (see Algorithm 6 in [11]) of the alternating descent method for inviscid Burgers equation is applicable to the viscous problem with small viscosity, and this method is efficient. 
ii) There exists another number $\nu_{1}$ such that if $\nu \in\left[\nu_{1}, \infty\right)$, then the solutions $u^{\nu}, \delta u^{\nu}$ are smooth for any $t>0$ and $p^{\nu}$ is smooth for $t<T$ and the thickness of the quasi-shock region is sufficiently big (say, bigger than the mesh size). In this case, if we replace the exact solution $p^{\nu}(x, 0)$ by $p(x, 0)$, then the error is probably not sufficiently small, thus the alternating descent method is not efficient for this case, instead the classical descent method is applicable.

iii) In the intermediate regime $\left(\nu_{0}, \nu_{1}\right)$, the method of asymptotic expansions does not work, whence the alternating descent method is not applicable. On the other hand, the classical descent method is not applicable either, since the quasi-shock region is so small that it cannot be detected numerically. Therefore, it is an open problem: How to design an efficient numerical scheme for optimal control of conservation laws in this case?

\section{Acknowledgement.}

This work was partly supported by the ERC Advanced Grant FP7-246775 NUMERIWAVES, the Grant PI2010-04 of the Basque Government, the ESF Research Networking Programme OPTPDE and Grant MTM2008-03541 of the MICINN, Spain. The research of $\mathrm{Ou}$ is also partially supported by the China Postdoctoral Science Foundation(Grant No. 201003077) and the Fundamental Research Funds for the Central Universities (Grant No. ZYGX2010J105). The authors would like to thank Prof. Zuazua for suggesting this problem, and Prof. Zhouping Xin, Prof. Zuazua and Prof. Castro for useful discussions.

\section{References}

[1] Bardos, C. and Pironneau, O. (2002) A formalism for the differentiation of conservation laws, C. R. Acad. Sci., Paris, Ser. I 335, 839-845.

[2] Bardos, C. and Pironneau, O. (2003) Derivatives and control in presence of shocks, Compu. Fluid Dyna. J., 11 No. 4, 383-392.

[3] Berger, M. and Fraenkel, L. E. (1970) On the asymptotic solution of a nonlinear Dirichlet problem, Journal of Math. mech, 19 No. 7, 553-585.

[4] Biagionit, H., and Obergugenberger, M. (1997) Generalized solutions to Burgers' equation, J. Diff. Eq., 97, 263-287.

[5] Biagionit, H., Cadeddu, L. and Cramchevs, T. (1997) Parabolic equations with conservative nonlinear term and singular initial data, Nonlinear Analysis TMA, 30, No. 4, 2489-2496.

[6] Bouchut, F. and James, F. (1998) One-dimensional transport equations with discontinuous coefficients, Nonlinear Anal. Th. Appl. 32, 891-933.

[7] Bouchut, F., James, F. and Mancini, S. (2005) Uniqueness and weak stability for multi-dimensional transport equations with one-sided Lipschitz coefficient, Ann. Sc. Norm. Super. Pisa Cl. Sci. 4, 1-25.

[8] Bressan, A. and Marson, A. (1995) A variational calculus for discontinuous solutions of systems of conservation laws, Commun. Partial Diff. Eqns. 20, 1491-1552.

[9] Bressan, A. and Marson, A. (1995) A maximum principle for optimally controlled systems of conservation laws, Rend. Sem. Mat. Univ. Padova 94, 79-94. 
[10] Brezis, H. and Friedman, A. (1983) Nonlinear parabolic equations involving measures as initial conditions, J. Math. Pures Appl. 62, 73-97.

[11] Castro, C., Palacios, F. and Zuazua, E. (2008) An alternating descent method for the optimal control of the inviscid Burgers equation in the presence of shocks, Math Models and Meth in Appl. Sci. 18, 369-416.

[12] Castro, C., Palacios, F. and Zuazua, E. (2010) Optimal control and vanishing viscosity for the Burgers equation. Integral Methods in Science and Engineering, Vol. 2. Computational Methods, Birkhauser-Boston, C. Constanda and M. E. Pérez eds., 65-90.

[13] Colombeau, J. and Langlais, M. (1990) An existence-uniqueness result for a nonlinear parabolic equation with Cauchy data distribution, J. Math. Anal. Appl. 145, 186-196.

[14] Duoandikoetxea, J. and Zuazua, E. (1992) Moments, masses de Dirac at décomposition de fonctions, C. R. Acad. Sci. Paris, 315, Série I, 693-698.

[15] Fife, P. (1988) Dynamics of Internal Layers and Diffusive Interfaces, CBMS-NSF regional conference series in applied mathematics, Society for Industrial and Applied Mathematics, Vol. 53.

[16] Fraenkel, L. E. (1969) On the method of matched asymptotic expansions, Proc. Camb. Phil. Soc., 65, Part I. A matching principle, 209-231, Part II. Some applications of the composite series, 233-261, Part III. Two boundary-value problems, 263-284.

[17] Fried, E. and Gurtin, M. (1994) Dynamic solid-solid transitions with phase characterized by an order parameter, Physica D 72, 287-308.

[18] Friedman, A. (1964) Partial Differential Equations of Parabolic Type, Prentice-Hall, Inc., Englewood Cliffs, New Jersy.

[19] Giles, M. and Pierce, N. (2001) Analytic adjoint solutions for the quasi onedimensional Euler equations, J. Fluid Mech. 426, 327-345.

[20] Godlewski, E. and Raviart, P. (1999) On the linearization of hyperbolic systems of conservation laws. A general nummerical approach, Math. Comput. Simul. 50, $77-95$.

[21] Goodman, J. and Xin, Z. (1992) Viscous limits for piecewise smooth solutions to systems of conservation laws, Arch. Rational Mech. Anal. 121, 235-265.

[22] Hinch, E. J. (1991) Perturbation Methods, Cambridge University Press.

[23] Holmes M. (1995) Introduction to Perturbation Methods, Springer-Verlag, New York.

[24] Hopf, E. (1950) The partial differential equation $u_{t}+u u_{x}=\mu u_{x x}$, Comm. Pure Appl. Math. 3, 201-230.

[25] Il'in A. M. (1992) Matching of Asymptotic Expansions of Solutins of Boundary Value Problems, Translations of Mathematical monographs, Vol. 102, American Math. Society, Providence, RI.

[26] James, F. and Sepúlveda, M. (1999) Convergence results for the flux identification in a scalar conservation law, SIAM J. Contr. Opti. 37, 869-891. 
[27] Kevorkian J. and Cole, J. (1996) Multiple Scale and Singular Perturbation Methods, Springer-Verlag, New York.

[28] Ladyzenskaya, O., Solonnikov, V. and Uralceva, N. (1968) Linear and Quasilinear Equations of Parabolic type, Trans. Math. Monographs, Vol. 23, American Math. Soc., Providence.

[29] LeVeque, R. (2002) Finite Volume Methods for Hyperbolic Problems, Combridge Univ. Press.

[30] Metivier, G. (2003) Stability of Multidimensional Shocks. Manuscript, Univ. de Rennes I.

[31] Pego, R. (1989) Front migration in the nonlinear Cahn-Hilliard equation, Proc. $R$. Soc. Lond. 422A, 261-278.

[32] Smoller, J. (1983) Shock waves and reaction-diffusion equations, Springer Verlag, New York.

[33] Ubrich, S. (2003) Adjoint-based derivative computations for the optimal control of discontinuous solutions of hyperbolic systems of conservation laws, Syst. Cont. Lett. 48, 313-328.

[34] Whitham, G. (1974) Linear and nonlinear waves, John Wiley \& Sons. 